\title{
Modelling the effects of condom use and antiretroviral therapy in controlling HIV/AIDS among heterosexuals, homosexuals and bisexuals
}

\author{
Noble Malunguza $^{\mathrm{a}}$, Steady Mushayabasa ${ }^{\mathrm{a}}$, Christinah Chiyaka ${ }^{\mathrm{ab}}$ and \\ Zindoga Mukandavire ${ }^{a b} *$ \\ ${ }^{a}$ Department of Applied Mathematics, National University of Science and Technology, Box AC 939 \\ Ascot, Bulawayo, Zimbabwe; ${ }^{b}$ Department of Public Health, China Medical University, \\ 91 Hsueh-Shih Road, Taichung, Taiwan 40402
}

(Received 8 March 2009; final version received 1 September 2009)

\begin{abstract}
A deterministic compartmental sex-structured HIV/AIDS model for assessing the effects of homosexuals and bisexuals in heterosexual settings in which homosexuality and bisexuality issues have remained taboo is presented. We extend the model to focus on the effects of condom use as a single strategy approach in HIV prevention in the absence of any other intervention strategies. Initially, we model the use of male condoms, followed by incorporating the use of both the female and male condoms. The model includes two primary factors in condom use to control HIV which are condom efficacy and compliance. Reproductive numbers for these models are computed and compared to assess the effectiveness of male and female condom use in a community. We also extend the basic model to consider the effects of antiretroviral therapy as a single strategy. The results from the study show that condoms can reduce the number of secondary infectives and thus can slow the development of the HIV/AIDS epidemic. Further, we note from the study that treatment of AIDS patients may enlarge the epidemic when the treatment drugs are not $100 \%$ effective and when treated AIDS patients indulge in risky sexual behaviour. Thus, the treatment with amelioration of AIDS patients should be accompanied with intense public health educational programs, which are capable of changing the attitude of treated AIDS patients towards safe sex. It is also shown from the study that the use of condoms in settings with the treatment may help in reducing the number of secondary infections thus slowing the epidemic.
\end{abstract}

Keywords: HIV/AIDS model; heterosexual; homosexual; bisexual; treatment; condoms

AMS Subject Classification: 34D05; 34D23; 92B05

\section{Introduction}

We begin by revisiting a deterministic compartmental sex-structured HIV/AIDS model for assessing the effects of homosexuals and bisexuals on the intrinsic dynamics of the disease in heterosexual settings in which homosexuality and bisexuality issues have remained taboo, partially studied in [18]. Due to criminalization of homosexual and bisexual activities in many countries in Africa and the lack of substantial research to map this high-risk population, it is not clear to what extent homosexual and bisexual activities have

*Corresponding author. Emails: zmukandavire@gmail.com; zmukandavire@nust.ac.zw 
contributed to the epidemic in this region and also to implement intervention strategies targeting such groups. Thus, in this paper, we extend the model in [18] to consider condom use and antiretroviral treatment as HIV/AIDS intervention strategies. The model subdivides the sexually active population at time $t$ into the following classes: $S_{\mathrm{f}}(t)$, susceptible female; $I_{\mathrm{f}}(t)$, infected female; $A_{\mathrm{f}}(t)$, female AIDS cases; $S_{\mathrm{m}_{1}}(t)$, susceptible heterosexual male population; $S_{\mathrm{m}_{2}}(t)$, susceptible homosexual male population; $I_{\mathrm{m}_{1}}(t)$, infected heterosexual male population; $I_{\mathrm{m}_{2}}(t)$, infected homosexual male population; $I_{\mathrm{m}_{3}}(t)$, infected bisexual male population; $A_{\mathrm{m}}(t)$, male AIDS cases. AIDS cases in the male and female populations are assumed to be sexually inactive, and so do not influence the dynamics of the virus. Individuals are referred to as male or female only in connection with features peculiar to their sex and in particular, the male to female infectivity rate is greater than that for female to male. The inclusion of male bisexuals in the model makes it suit most heterosexual settings (especially in Africa) in the sense that in these settings most of the homosexuals resort to bisexuality due to the fact that homosexual and bisexual activities are regarded as taboo, thus by so doing homosexuals will also not meet denial in the community. The population is assumed to be uniform and homogeneously mixed. The total population is given by

$$
N(t)=S_{\mathrm{f}}(t)+I_{\mathrm{f}}(t)+A_{\mathrm{f}}(t)+S_{\mathrm{m}_{1}}(t)+S_{\mathrm{m}_{2}}(t)+I_{\mathrm{m}_{1}}(t)+I_{\mathrm{m}_{2}}(t)+I_{\mathrm{m}_{3}}(t)+A_{\mathrm{m}}(t) .
$$

It is assumed that, at any moment in time, new recruits enter the sexually active population at a rate $\Lambda$. A proportion $\pi_{0}$ of these individuals are assumed to be female susceptibles (categorized in the $S_{\mathrm{f}}$ class) and the complementary proportion $\left(1-\pi_{0}\right)$, are male susceptibles and categorized in the male susceptible classes $S_{\mathrm{m}_{1}}(t)$ and $S_{\mathrm{m}_{2}}(t)$ with proportions $\pi_{1}$ and $\pi_{2}$, respectively, thus $\pi_{0}+\pi_{1}+\pi_{2}=1$. Female susceptibles acquire infection at a time-dependent rate

$$
\lambda_{\mathrm{f}}=\frac{\beta_{\mathrm{m}} c_{\mathrm{f}}\left\{I_{\mathrm{m}_{1}}(t)+\eta I_{\mathrm{m}_{3}}(t)\right\}}{N_{\mathrm{m}}(t)} .
$$

The force of infection $\lambda_{\mathrm{f}}$ depends on the probability that an infected male infects his female sexual partner $\beta_{\mathrm{m}}$, the rate at which a female acquires new sexual partners per unit time $c_{\mathrm{f}}$ and the proportion of infected males in each category $\left(I_{\mathrm{m}_{1}}\right.$ and $\left.I_{\mathrm{m}_{3}}\right)$. The enhancement factor, $\eta>1$ models the relative infectiousness of the purely heterosexual individuals to bisexuals $[14,15]$. Here, we assume that a group of bisexuals are more at risk because they are part of a high-risk homosexual group and further, due to the fact that homosexuality and bisexuality issues have remained taboo in African heterosexual settings. To date, no intervention strategies have been implemented to target or address such groups. In (1), the total sexually active heterosexual and bisexual population is given by $N_{\mathrm{m}}=S_{\mathrm{m}_{1}}(t)+I_{\mathrm{m}_{1}}(t)+I_{\mathrm{m}_{3}}(t)$. Heterosexual male susceptibles acquire infection at a time-dependent rate

$$
\lambda_{\mathrm{m}_{1}}=\beta_{\mathrm{f}} c_{\mathrm{m}_{1}} \frac{I_{\mathrm{f}}(t)}{N_{\mathrm{f}}(t)} .
$$

In (2), $\beta_{\mathrm{f}}$ is the probability that an infected female infects her male sexual partner, $c_{\mathrm{m}_{1}}$ is the rate at which heterosexually active male population acquires new sexual partners per unit time and $N_{\mathrm{f}}(t)=S_{\mathrm{f}}(t)+I_{\mathrm{f}}(t)$ denotes the sexually interacting female population. Upon infection, a proportion $\delta_{1}$ of the heterosexually infected male population moves to class $I_{\mathrm{m}_{1}}$ of purely heterosexual active infected male population and the complementary 
proportion, $1-\delta_{1}$ moves to class $I_{\mathrm{m}_{3}}$ of male bisexuals. Homosexual male population acquires infection at a time-dependent rate

$$
\lambda_{\mathrm{m}_{2}}=\beta_{\mathrm{h}} c_{\mathrm{m}_{2}} \frac{I_{\mathrm{m}_{2}}(t)}{N_{\mathrm{m}_{2}}(t)},
$$

where $\beta_{\mathrm{h}}$ is the probability that an infected homosexual infects his sexual partner, $c_{\mathrm{m}_{2}}$ is the rate at which heterosexually active homosexual population acquires new sexual partners per unit time and $N_{\mathrm{m}_{2}}(t)=S_{\mathrm{m}_{2}}(t)+I_{\mathrm{m}_{2}}(t)$ denotes the total sexually interacting homosexual population. Upon infection, a proportion, $\delta_{2}$, of the homosexually infected male population moves to class $I_{\mathrm{m}_{2}}$ of purely homosexual active infected male population and the complementary proportion, $1-\delta_{2}$ moves to class $I_{\mathrm{m}_{3}}$ of male bisexuals. For simplicity, the model does not explicitly consider a category for susceptible bisexuals $\left(S_{\mathrm{m}_{3}}\right)$ and it assumes that bisexual infectives only arise when a certain proportion of heterosexuals and homosexuals become bisexuals upon HIV infection.

There is a constant emigration rate $\alpha>0$ of individuals to other countries except for the AIDS patients. This assumption makes the model more appropriate for African settings (especially Zimbabwe) where a significant proportion of the population emigrates to developed countries for better educational facilities and in search of employment. HIV infected individuals progress to AIDS stage at a constant rate, $\gamma>0$. The natural death rate is assumed to be proportional to the number in each class, $\mu>0$. AIDS patients have an additional disease-induced mortality rate, $\nu>0$ and are easily recognized in the population, and thus are not a threat in the spread of the epidemic. The model flow diagram

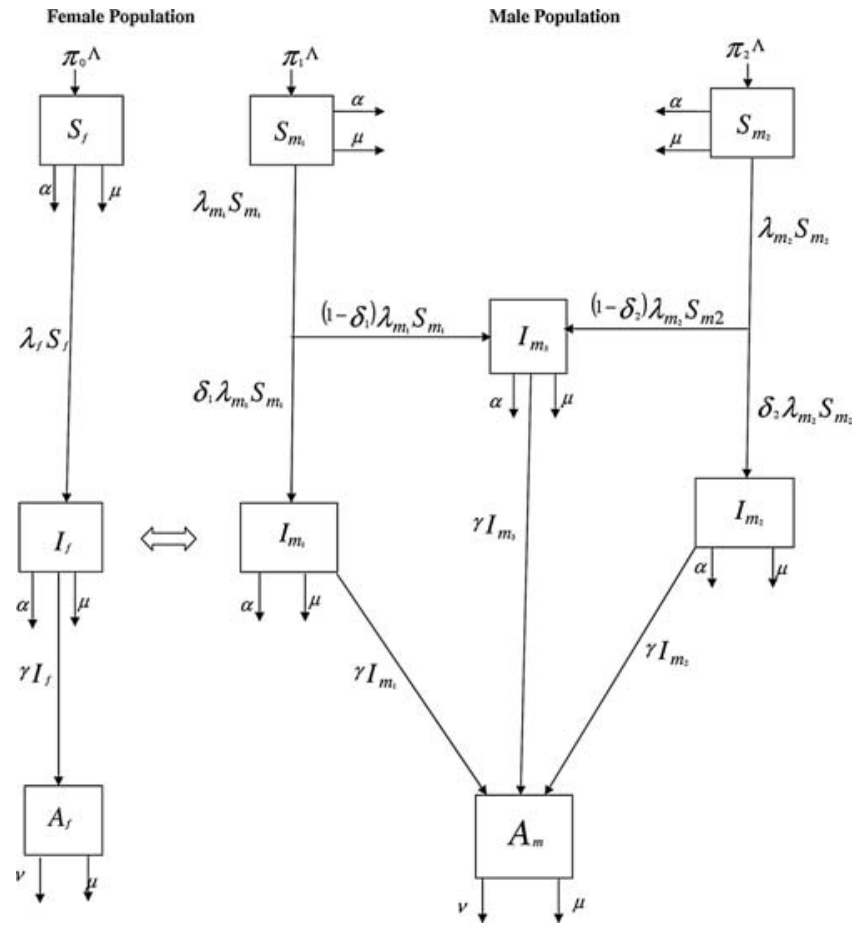

Figure 1. Model flow diagram. 
is shown in Figure 1 . The arrow $(\Leftrightarrow)$ in Figure 1 denotes sexual interaction of the male and female populations.

Putting the formulations and the assumptions together gives the following system of differential equations,

$$
\begin{gathered}
d \frac{S_{\mathrm{f}}}{\mathrm{d} t}=\pi_{0} \Lambda-\lambda_{\mathrm{f}} S_{\mathrm{f}}(t)-(\alpha+\mu) S_{\mathrm{f}}(t) \\
d \frac{I_{\mathrm{f}}}{\mathrm{d} t}=\lambda_{\mathrm{f}} S_{\mathrm{f}}(t)-(\gamma+\alpha+\mu) I_{\mathrm{f}}(t) \\
d \frac{A_{\mathrm{f}}}{\mathrm{d} t}=\gamma I_{\mathrm{f}}(t)-(\nu+\mu) A_{\mathrm{f}}(t) \\
d \frac{S_{\mathrm{m}_{1}}}{\mathrm{~d} t}=\pi_{1} \Lambda-\lambda_{\mathrm{m}_{1}} S_{\mathrm{m}_{1}}(t)-(\alpha+\mu) S_{\mathrm{m}_{1}}(t) \\
d \frac{S_{\mathrm{m}_{2}}}{\mathrm{~d} t}=\pi_{2} \Lambda-\lambda_{\mathrm{m}_{2}} S_{\mathrm{m}_{2}}(t)-(\alpha+\mu) S_{\mathrm{m}_{2}}(t) \\
d \frac{I_{\mathrm{m}_{1}}}{\mathrm{~d} t}=\delta_{1} \lambda_{\mathrm{m}_{1}} S_{\mathrm{m}_{1}}(t)-(\gamma+\alpha+\mu) I_{\mathrm{m}_{1}}(t) \\
d \frac{I_{\mathrm{m}_{2}}}{\mathrm{~d} t}=\delta_{2} \lambda_{\mathrm{m}_{2}} S_{\mathrm{m}_{2}}(t)-(\gamma+\alpha+\mu) I_{\mathrm{m}_{2}}(t) \\
d \frac{I_{\mathrm{m}_{3}}}{\mathrm{~d} t}=\left(1-\delta \lambda_{1}\right) \lambda_{\mathrm{m}_{1}} S_{\mathrm{m}_{1}}(t)+\left(1-\delta_{2}\right) \lambda_{\mathrm{m}_{2}} S_{\mathrm{m}_{2}}(t)-(\gamma+\alpha+\mu) I_{\mathrm{m}_{3}}(t) \\
d \frac{A A_{\mathrm{m}}}{\mathrm{d} t}=\gamma\left(I_{\mathrm{m}_{1}}(t)+I_{\mathrm{m}_{2}}(t)+I_{\mathrm{m}_{3}}(t)\right)-(\mu+\nu) A_{\mathrm{m}}(t)
\end{gathered}
$$

The mathematical properties of model system (4) including stabilities of equilibria are studied in [18]. The disease-free equilibrium (DFE) for model system (4) is,

$$
\begin{aligned}
\mathcal{E}^{0} & =\left(S_{\mathrm{f}}^{0}, S_{\mathrm{m}_{1}}^{0}, S_{\mathrm{m}_{2}}^{0}, I_{\mathrm{f}}^{0}, I_{\mathrm{m}_{1}}^{0}, I_{\mathrm{m}_{2}}^{0}, I_{\mathrm{m}_{3}}^{0}, A_{\mathrm{f}}^{0}, A_{m}^{0}\right) \\
& =\left(\frac{\pi_{0} \Lambda}{\alpha+\mu}, \frac{\pi_{1} \Lambda}{\alpha+\mu}, \frac{\pi_{2} \Lambda}{\alpha+\mu}, 0,0,0,0,0,0\right) .
\end{aligned}
$$

The linear stability of $\mathcal{E}^{0}$ is governed by the basic reproductive number $\mathcal{R}_{0}[1,6,9,12]$. The stability of this equilibrium will be investigated using the next generation operator $[10,20]$. Using the notation in [20] on the model system (4), the matrices $\mathbf{F}$ and $\mathbf{V}$, for the new infection terms and the remaining transfer terms are, respectively, given by

$$
\mathbf{F}=\left(\begin{array}{cccc}
0 & \frac{\pi_{0} \beta_{\mathrm{m}} c_{\mathrm{f}}}{\pi_{1}} & 0 & \frac{\pi_{0} \eta \beta_{\mathrm{m}} c_{\mathrm{f}}}{\pi_{1}} \\
\frac{\delta_{1} \pi_{1} \beta_{\mathrm{f}} c_{\mathrm{m}_{1}}}{\pi_{0}} & 0 & 0 & 0 \\
0 & 0 & \delta_{2} \beta_{\mathrm{h}} c_{\mathrm{m}_{2}} & 0 \\
\frac{\left(1-\delta_{1}\right) \pi_{1} \beta_{\mathrm{f}} c_{\mathrm{m}_{1}}}{\pi_{0}} & 0 & \left(1-\delta_{2}\right) \beta_{\mathrm{h}} c_{\mathrm{m}_{2}} & 0
\end{array}\right)
$$


and

$$
\mathbf{V}=\left(\begin{array}{cccc}
\alpha+\gamma+\mu & 0 & 0 & 0 \\
0 & \alpha+\gamma+\mu & 0 & 0 \\
0 & 0 & \alpha+\gamma+\mu & 0 \\
0 & 0 & 0 & \alpha+\gamma+\mu
\end{array}\right)
$$

It follows that the basic reproduction number for model system (4), denoted by $\mathcal{R}_{0}$ is given by

$$
\mathcal{R}_{0}=\rho\left(\mathbf{F} \mathbf{V}^{-1}\right)=\max \left\{\frac{\delta_{2} \beta_{\mathrm{h}} c_{\mathrm{m}_{2}}}{\alpha+\gamma+\mu}, \sqrt{\frac{\beta_{\mathrm{f}} \beta_{\mathrm{m}} c_{\mathrm{f}} c_{\mathrm{m}_{1}}}{(\alpha+\gamma+\mu)^{2}} f\left(\delta_{1}, \eta\right)}\right\}=\max \left\{\mathcal{R}_{01}, \mathcal{R}_{02}\right\}
$$

where $f\left(\delta_{1}, \eta\right)=\delta_{1}+\eta\left(1-\delta_{1}\right)$ measures the combined effects of homosexuality and bisexuality in heterosexual settings. Next, we extend model system (4) to incorporate condom use. The basic reproduction number $\left(\mathcal{R}_{0}\right)$ measures the average number of new infections generated by a single infected individual in a completely susceptible population. The partial reproductive number $\mathcal{R}_{01}$ (homosexuality-induced reproductive number) defines the number of secondary infections due to purely homosexual transmission and $\mathcal{R}_{02}$ (heterosexuality and bisexuality-induced reproductive number) defines the number of secondary infections due to heterosexual and bisexual transmission.

\section{Condom use}

\subsection{Male condom use}

A male condom is a sheath worn on an erect penis to prevent the exchange of body fluids during sexual intercourse. One disadvantage of the male condom lies in the fact that, by definition, men control its use. Effectiveness of the condoms has been the subject of several studies; most of these studies portray that the proper and consistent use of condoms during sexual intercourse results in an $80 \%$ reduction in HIV incidence, a level slightly less than for pregnancy [17]. Model system (4) is extended to incorporate male condom use. We assume that the male condom is used by heterosexuals, homosexuals and bisexuals as the only intervention strategy to prevent the spread of the epidemic. The parameter $\sigma_{\mathrm{m}}$ models male condom use and by considering this intervention for model system (4), female susceptibles acquire infection at a time-dependent rate

$$
\lambda_{\mathrm{f}}=\frac{\sigma_{\mathrm{m}} \beta_{\mathrm{m}} c_{\mathrm{f}}\left\{I_{\mathrm{m}_{1}}(t)+\eta I_{\mathrm{m}_{3}}(t)\right\}}{N_{\mathrm{m}}(t)}
$$

heterosexual male susceptibles acquire infection at a time-dependent rate

$$
\lambda_{\mathrm{m}_{1}}=\sigma_{\mathrm{m}} \beta_{\mathrm{f}} c_{\mathrm{m}_{1}} \frac{I_{\mathrm{f}}(t)}{N_{\mathrm{f}}(t)}
$$

and homosexual male population acquire infection at a time-dependent rate

$$
\lambda_{\mathrm{m}_{2}}=\sigma_{\mathrm{m}} \beta_{\mathrm{h}} c_{\mathrm{m}_{2}} \frac{I_{\mathrm{m}_{2}}(t)}{N_{\mathrm{m}_{2}}(t)} .
$$


The parameter $\sigma_{\mathrm{m}}=1-\epsilon_{\mathrm{m}} \omega_{\mathrm{m}}$, where $\epsilon_{\mathrm{m}}$ and $\omega_{\mathrm{m}}$ denote the male condom efficacy and compliance, respectively $[16,17]$. We define the product $p_{\mathrm{m}}=\epsilon_{\mathrm{m}} \omega_{\mathrm{m}}$ as the condominduced preventability of HIV transmission per year [17]. Thus, the per exposure risk of infection, say $\Gamma_{\mathrm{c}}$, becomes;

$$
\Gamma_{\mathrm{c}}=\beta_{i}\left(1-\epsilon_{\mathrm{m}} \omega_{\mathrm{m}}\right) \quad \text { for } i=f \text { or } m
$$

and $\beta_{i}$ is the exposure risk of infection when no protection of any kind is used. The male condom-induced reproductive number $\mathcal{R}_{\mathrm{m}}$ becomes,

$$
\begin{aligned}
\mathcal{R}_{\mathrm{m}} & =\max \left\{\frac{\sigma_{\mathrm{m}} \delta_{2} \beta_{\mathrm{h}} c_{\mathrm{m}_{2}}}{\alpha+\gamma+\mu}, \sigma_{\mathrm{m}} \sqrt{\frac{\beta_{\mathrm{f}} \beta_{\mathrm{m}} c_{\mathrm{f}} c_{\mathrm{m}_{1}}}{(\alpha+\gamma+\mu)^{2}}\left[\delta_{1}+\eta\left(1-\delta_{1}\right)\right]}\right\} \\
& =\max \left\{\sigma_{\mathrm{m}} \mathcal{R}_{01}, \sigma_{\mathrm{m}} \mathcal{R}_{02}\right\}=\max \left\{\mathcal{R}_{\mathrm{m}_{1}}, \mathcal{R}_{\mathrm{m}_{2}}\right\}
\end{aligned}
$$

The male condom-induced reproductive number $\left(\mathcal{R}_{\mathrm{m}}\right)$ can be presented as,

$$
\mathcal{R}_{\mathrm{m}}\left(\epsilon_{\mathrm{m}}, \omega_{\mathrm{m}}\right)=\left(1-\epsilon_{\mathrm{m}} \omega_{\mathrm{m}}\right) \mathcal{R}_{0} .
$$

The above expression emphasizes the role of efficacy and compliance of a male condom in preventing HIV transmission. We also observe that $\mathcal{R}_{\mathrm{m}}\left(0, \omega_{\mathrm{m}}\right)=\mathcal{R}_{\mathrm{m}}\left(\epsilon_{\mathrm{m}}, 0\right)=$ $\mathcal{R}_{\mathrm{m}}(0,0)=\mathcal{R}_{0}$ and that $\mathcal{R}_{\mathrm{m}}\left(\epsilon_{\mathrm{m}}, \omega_{\mathrm{m}}\right) \leq \mathcal{R}_{0}$ for all $\epsilon_{\mathrm{m}}, \omega_{\mathrm{m}} \geq 0$. Thus, the basic reproductive number (reproductive number in the absence of male condoms and any other interventions) $\mathcal{R}_{0}$ is greater than the male condom-induced reproductive number, $\left(\mathcal{R}_{0}>\mathcal{R}_{\mathrm{m}}\right)$. Thus, the use of male condoms as a prevention strategy can reduce the spread of HIV in a community.

In an analogous fashion, to the analysis used in [17], we state and prove Lemma 1.

LEMMA 1. HIV can be eradicated from the community if the preventability $p_{\mathrm{m}}=\epsilon_{\mathrm{m}} \omega_{\mathrm{m}}$ exceeds the threshold value $p_{\mathrm{mc}}$.

Proof.

$$
\mathcal{R}_{\mathrm{m}}\left(\epsilon_{\mathrm{m}}, \omega_{\mathrm{m}}\right)=\left(1-\epsilon_{\mathrm{m}} \omega_{\mathrm{m}}\right) \mathcal{R}_{0}, \quad \epsilon_{\mathrm{mc}}=\frac{1}{\omega_{\mathrm{m}}}\left(1-\frac{1}{\mathcal{R}_{0}}\right)
$$

then $\mathcal{R}\left(\epsilon_{\mathrm{mc}}\right)=1$ assuming $\omega_{\mathrm{m}}>0$. If

$$
\omega_{\mathrm{mc}}=\frac{1}{\epsilon_{\mathrm{m}}}\left(1-\frac{1}{\mathcal{R}_{0}}\right)
$$

then $\mathcal{R}_{\mathrm{m}}\left(\omega_{\mathrm{mc}}\right)=1$, assuming $\epsilon_{\mathrm{m}}>0$. Since $\mathcal{R}_{\mathrm{m}}$ is a decreasing function of both $\epsilon_{\mathrm{m}}$ and $\omega_{\mathrm{m}}$, it follows that $\mathcal{R}_{\mathrm{m}}<1$ whenever $\epsilon_{\mathrm{m}}>\epsilon_{\mathrm{mc}}$ or $\omega_{\mathrm{m}}>\omega_{\mathrm{mc}}$. In addition, it is also essential to note that $\mathcal{R}_{\mathrm{m}}$ is also a decreasing function of $p_{\mathrm{m}}$. This implies that HIV can be eradicated in the community if $p_{\mathrm{m}}>p_{\mathrm{mc}}=\epsilon_{\mathrm{mc}} \omega_{\mathrm{mc}}$.

\subsection{Effects of male condom use on $\mathcal{R}_{0}$}

The male condom-induced reproductive number $\mathcal{R}_{\mathrm{m}}$ is expressed in terms of $\mathcal{R}_{0}$. Thus, we write $\mathcal{R}_{\mathrm{m}}=\mathcal{R}_{\mathrm{m}}\left(\mathcal{R}_{0}\right)$ to emphasize the dependence of $\mathcal{R}_{\mathrm{m}}$ on $\mathcal{R}_{0}$. The obtained results in 


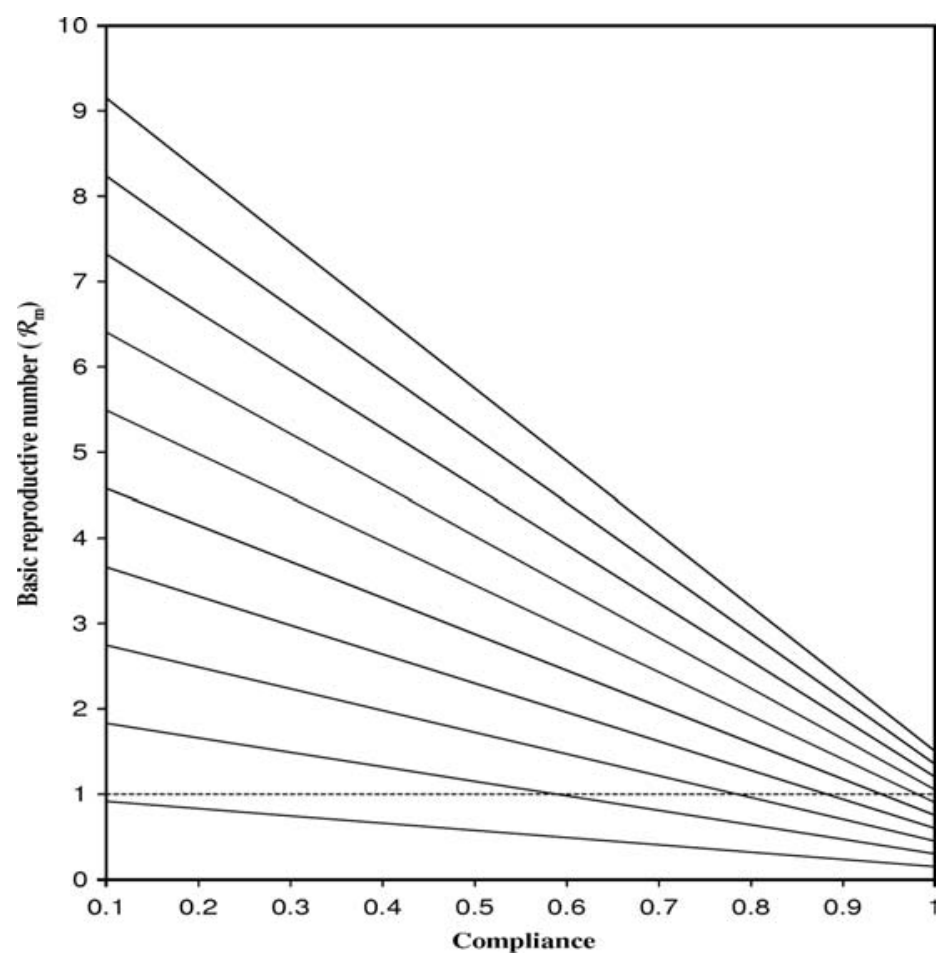

Figure 2. Simulation results showing trends of $\mathcal{R}_{\mathrm{m}}$ against compliance $\omega_{\mathrm{m}}$. The corresponding values of the reproductive number $\mathcal{R}_{\mathrm{m}}$ are obtained by varying $\mathcal{R}_{0}$ from 1 to 10 in step 1 and $\omega_{\mathrm{m}}$ from 0.1 to 1 in the step 0.1. Efficacy $\epsilon_{\mathrm{m}}=0.85$. The dashed line is $\mathcal{R}_{\mathrm{m}}=1$. The illustration shows that there are certain values of $\mathcal{R}_{0}$ at which the introduction of male condoms will not reduce $\mathcal{R}_{\mathrm{m}}$ to values below unity even if compliance of $100 \%$ is achieved.

Figure 2 illustrate that there are certain values for $\mathcal{R}_{0}$ at which the introduction of male condoms may not reduce $\mathcal{R}_{\mathrm{m}}$ to values below unity, even if $100 \%$ condom compliance is achieved. Further, for the values of $\mathcal{R}_{0}$ which $\mathcal{R}_{\mathrm{m}}$ can be reduced to values below one $\left(\mathcal{R}_{\mathrm{m}}<1\right)$, the compliance is high $\left(\omega_{\mathrm{m}}>90 \%\right)$ and perhaps unattainable for high-values of $\mathcal{R}_{0}$ suggesting that for such a population, HIV/AIDS may not be controlled using male condoms alone. Note that in our computations of the values of $\mathcal{R}_{\mathrm{m}}$, we vary $\mathcal{R}_{0}$ to obtain the corresponding values of $\mathcal{R}_{\mathrm{m}}$, which we plot against compliance.

\subsection{Male and female condoms use}

A female condom is a loose-fitting polyurethane sheath with a flexible sheath at either end. The inner, closed ring is pushed into the vagina, while the outer, open ring rests outside the vagina. The female condom came into existence when it became clear that women were both biologically and socioculturally more vulnerable to HIV infection [16,17]. We extend the model system (4) to model the effect of both the male and female condoms in a community as the only intervention strategy. The parameter $\sigma_{\mathrm{fm}}$ models the effect of female and male condom use. We note that the introduction of the female condom in a setting with male condom use will benefit the other classes in the population except the homosexuals. Thus, in this section, we will focus on the effects of the female condom on the male condom heterosexuality and bisexuality-induced partial reproductive number 
$\left(\mathcal{R}_{\mathrm{m}_{2}}\right)$. The rate of infection for different classes will be represented as follows, female susceptibles acquire infection at a time-dependent rate

$$
\lambda_{\mathrm{f}}=\frac{\sigma_{\mathrm{fm}} \beta_{\mathrm{m}} c_{\mathrm{f}}\left\{I_{\mathrm{m}_{1}}(t)+\eta I_{\mathrm{m}_{3}}(t)\right\}}{N_{\mathrm{m}}(t)}
$$

heterosexual male susceptibles acquire infection at a time-dependent rate

$$
\lambda_{\mathrm{m}_{1}}=\sigma_{\mathrm{fm}} \beta_{\mathrm{f}} c_{\mathrm{m}_{1}} \frac{I_{\mathrm{f}}(t)}{N_{\mathrm{f}}(t)},
$$

where the parameter $\sigma_{\mathrm{fm}}=\sigma_{\mathrm{f}} \sigma_{\mathrm{m}}=\left(1-\epsilon_{\mathrm{f}} \omega_{\mathrm{f}}\right)\left(1-\epsilon_{\mathrm{m}} \omega_{\mathrm{m}}\right)$. We assume that the use of female and male condoms confer protection independently. The parameters $\sigma_{\mathrm{f}}$ and $\sigma_{\mathrm{m}}$ measure the effectiveness of both the female and male condoms in reducing the probability of transmission $\beta_{i}$ for $i=f, m$. We define the condom efficacy as $\epsilon_{i}$ and condom compliance as $\omega_{i}$ for $i=f, m$. The parameters $\omega_{\mathrm{m}}$ and $\omega_{\mathrm{f}}$ model the compliance of the male and female condoms, respectively. If the male and female condoms are used correctly and consistently, the exposure risk of infection becomes,

$$
\Gamma_{\mathrm{c}}=\beta_{i}\left(1-\left(\epsilon_{\mathrm{f}} \omega_{\mathrm{f}}+\epsilon_{\mathrm{m}} \omega_{\mathrm{m}}-\epsilon_{\mathrm{f}} \epsilon_{\mathrm{m}} \omega_{\mathrm{f}} \omega_{\mathrm{m}}\right)\right) \quad \text { for } i=f \text { or } m .
$$

The male and female condoms heterosexuality and bisexuality-induced partial reproductive number becomes,

$$
\mathcal{R}_{\mathrm{fm} 2}=\sigma_{\mathrm{fm}} \sqrt{\frac{\beta_{\mathrm{f}} \beta_{\mathrm{m}} c_{\mathrm{f}} c_{\mathrm{m}_{1}}}{(\alpha+\gamma+\mu)^{2}}\left[\delta_{1}+\eta\left(1-\delta_{1}\right)\right]}=\sigma_{\mathrm{fm}} \mathcal{R}_{02} .
$$

$\mathcal{R}_{\mathrm{fm}_{2}}$ measures the average number of new infections generated by a single infected heterosexual or bisexual infected individual in a completely susceptible population. Analysis of the reproductive number shows that HIV/AIDS can be reduced through the use of both the condoms since $\mathcal{R}_{\mathrm{fm}_{2}}<\mathcal{R}_{\mathrm{m}_{2}}<\mathcal{R}_{02}$.

The male and female condoms heterosexuality and bisexuality-induced partial reproductive number $\mathcal{R}_{\mathrm{fm}}$ can be presented as,

$$
\begin{aligned}
\mathcal{R}_{\mathrm{fm}}\left(\epsilon_{\mathrm{f}}, \epsilon_{\mathrm{m}} \omega_{\mathrm{f}}, \omega_{\mathrm{m}}\right) & =\left(1-\left(\epsilon_{\mathrm{f}} \omega_{\mathrm{f}}+\epsilon_{\mathrm{m}} \omega_{\mathrm{m}}-\epsilon_{\mathrm{f}} \epsilon_{\mathrm{m}} \omega_{\mathrm{f}} \omega_{\mathrm{m}}\right)\right) \mathcal{R}_{02} \\
& =\mathcal{R}_{\mathrm{m}_{2}}-\left(\epsilon_{\mathrm{f}} \omega_{\mathrm{f}}-\epsilon_{\mathrm{f}} \epsilon_{\mathrm{m}} \omega_{\mathrm{f}} \omega_{\mathrm{m}}\right) \mathcal{R}_{02}
\end{aligned}
$$

where $\mathcal{R}_{02}$ is the basic heterosexuality and bisexuality-induced partial reproductive number and $\mathcal{R}_{\mathrm{m}_{2}}$ is the male condom heterosexuality and bisexuality-induced partial reproductive number. We also note that

$$
\mathcal{R}_{\mathrm{fm}_{2}}\left(0, \epsilon_{\mathrm{m}}, \omega_{\mathrm{f}}, \omega_{\mathrm{m}}\right)=\mathcal{R}_{\mathrm{fm}_{2}}\left(\epsilon_{\mathrm{f}}, \epsilon_{\mathrm{m}}, 0, \omega_{\mathrm{m}}\right)=\mathcal{R}_{\mathrm{fm}}\left(0, \epsilon_{\mathrm{m}}, 0, \omega_{\mathrm{m}}\right)=\mathcal{R}_{02}
$$

and $\mathcal{R}_{\mathrm{fm}_{2}}<\mathcal{R}_{\mathrm{m}_{2}}$ for all $\epsilon_{\mathrm{f}}, \omega_{\mathrm{f}}, \omega_{\mathrm{m}}, \epsilon_{\mathrm{m}}>0$. Thus, we can safely deduce that $\mathcal{R}_{\mathrm{fm}_{2}}<$ $\mathcal{R}_{\mathrm{m}_{2}}<\mathcal{R}_{02}$ for all $\epsilon_{i}, \omega_{i}$ for $i=f, m$ which clearly shows that the proper and consistent use of both the male and female condoms in the community would help in containing the HIV/AIDS epidemic much better than the use of only the male condom among heterosexuals and bisexuals.

In Figure 3, we illustrate the relationship and behaviour of $\mathcal{R}_{01}, \mathcal{R}_{02}, \mathcal{R}_{\mathrm{m}_{1}}, \mathcal{R}_{\mathrm{m}_{2}}$ and $\mathcal{R}_{\mathrm{fm}_{2}}$ for increasing number of sexual partners per unit time $\left(c_{\mathrm{m}_{1}}=c_{\mathrm{m}_{2}}=c_{\mathrm{f}}\right)$. The 


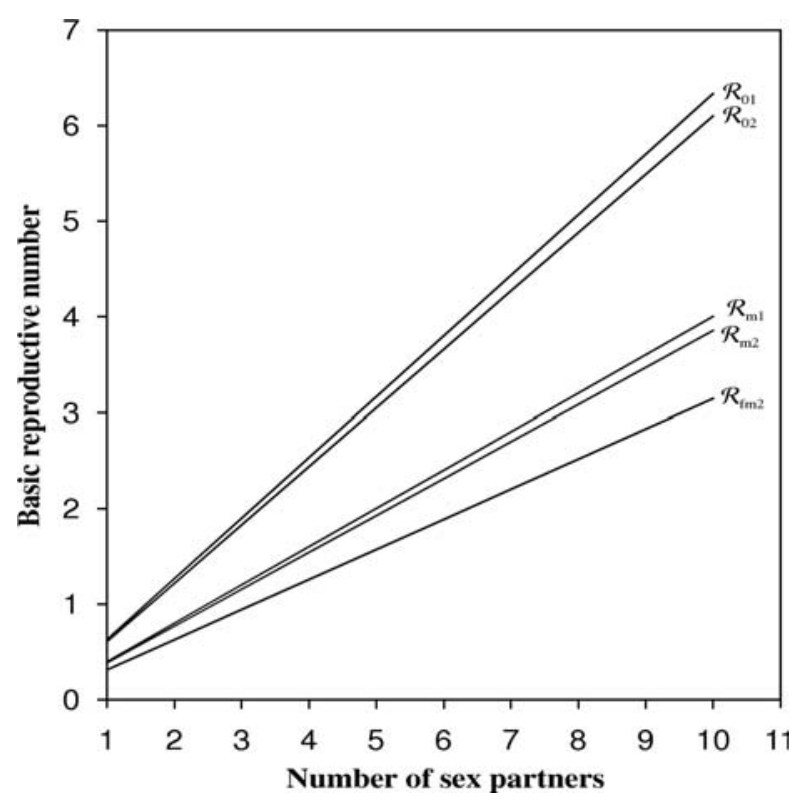

Figure 3. Simulation results showing trends of the reproductive numbers $\mathcal{R}_{01}, \mathcal{R}_{02}, \mathcal{R}_{\mathrm{m}_{1}}, \mathcal{R}_{\mathrm{m}_{2}}, \mathcal{R}_{\mathrm{fm}_{2}}$ for varying number of sexual partners. Sexual partners are varied from 1 to 10 per unit time. Parameters used are in Table 1. The trends show the relationship and behaviour of $\mathcal{R}_{01}, R_{02}, \mathcal{R}_{\mathrm{m}_{1}}, \mathcal{R}_{\mathrm{m}_{2}}$ and $\mathcal{R}_{\mathrm{fm}_{2}}$ for increasing the number of sexual partners.

illustration shows that $\mathcal{R}_{\mathrm{m}_{1}}<\mathcal{R}_{01}$ and $\mathcal{R}_{\mathrm{fm}_{2}}<\mathcal{R}_{\mathrm{m}_{2}}<\mathcal{R}_{02}$ remains the relationship for the reproductive numbers as obtained from the analytic approach when male condom only is presented and when both the female and male condoms are presented. The results further confirm that the use of both the female and male condoms would benefit the heterosexuals and bisexuals in the community much more than the use of the male condom only since $\mathcal{R}_{\mathrm{fm}_{2}}<\mathcal{R}_{\mathrm{m}_{2}}$ for all $\epsilon_{i}, \omega_{i}>0$ for $i=f, m$.

\subsection{Numerical simulations}

Numerical simulations of the extension of model system (4) with condom use are carried out using a set of parameter values given in Table 1. We use a fourth order Runge-Kutta numerical scheme coded in $\mathrm{C}^{++}$programming language for the numerical simulations.

Figure 4 shows a decrease in the number of infectives for homosexuals, heterosexuals and bisexuals when male and female condoms compliance increases from 0.1 to 1 . The results show that when compliance increases the number of infectives decreases and this reduces HIV/AIDS prevalence in a community if male and female condoms are used correctly and consistently by a large proportion of infectives.

\section{Treatment}

We extend model system (4) to incorporate the treatment of AIDS patients, in which we consider the treatment to be given to AIDS individuals who are ill and have experienced AIDS-defining symptoms, or whose CD $4+\mathrm{T}$ cell count is below $200 \mu \mathrm{l}^{-1}$, which is the recommended AIDS defining stage [21]. It is assumed that treated AIDS patients are capable of sexual interaction with the rest of the community. Now the model will include the following classes: treated female population $A_{\mathrm{tf}}$, treated heterosexual male population 
Table 1. Data for the HIV/AIDS models.

\begin{tabular}{|c|c|c|c|}
\hline Parameter & Symbol & Value & Source \\
\hline Recruitment rate & $\Lambda$ & $100,000 /$ year & [18] \\
\hline Proportion of mature females & $\pi_{0}$ & 0.5 & [18] \\
\hline $\begin{array}{l}\text { Proportion of mature } \\
\text { heterosexual male }\end{array}$ & $\pi_{1}$ & 0.4 & [18]] \\
\hline Proportion of mature homosexuals & $\pi_{2}$ & 0.1 & [18] \\
\hline $\begin{array}{l}\text { Proportion of purely } \\
\text { (heterosexuals, homosexuals) }\end{array}$ & $\left(\delta_{1}, \delta_{2}\right)$ & $(0.8,0.4)$ & [18] \\
\hline Natural death rate & $\mu$ & $0.02 /$ year & {$[16,17]$} \\
\hline AIDS-related death rate & $v$ & $0.333 /$ year & {$[16,17]$} \\
\hline Emigration rate & $\alpha$ & $0.01 /$ year & {$[16,17]$} \\
\hline $\begin{array}{l}\text { Rate of progression to } \\
\text { AIDS }\end{array}$ & $\gamma$ & 8/year & {$[16,17]$} \\
\hline Probability of transmission & $\left(\beta_{\mathrm{f}}, \beta_{\mathrm{m}}, \beta_{\mathrm{h}}\right)$ & $(0.0497,0.1637,0.2456)$ & {$[4,5,16-18]$} \\
\hline $\begin{array}{l}\text { Rate of acquiring sexual } \\
\text { partners }\end{array}$ & $\left(c_{\mathrm{f}}, c_{\mathrm{m}_{1}}, c_{\mathrm{m}_{2}}\right)$ & $(3,3,3)$ partners/year & {$[16-18]$} \\
\hline Enhancement factor & $\eta$ & 1.5 & [18] \\
\hline Condom efficacy & $\left(\epsilon_{\mathrm{f}}=\epsilon_{\mathrm{m}}\right)$ & 0.92 & {$[16,17,19]$} \\
\hline Condom compliance & $\left(\omega_{\mathrm{f}}=\omega_{\mathrm{m}}\right)$ & $(0.2,0.4)$ & [17] \\
\hline The basic reproductive number & $\left(\mathcal{R}_{01}, \mathcal{R}_{02}\right)$ & $(1.901,1.8312)$ & \\
\hline The reproductive numbers & $\left(\mathcal{R}_{\mathrm{m}_{1}}, \mathcal{R}_{\mathrm{m}_{2}}, \mathcal{R}_{\mathrm{fm}_{2}}\right)$ & $(1.2014,1.1573,0.9490)$ & \\
\hline
\end{tabular}

$A_{\mathrm{tm}_{1}}$, treated homosexual male population $A_{\mathrm{tm}_{2}}$ and treated bisexual male population $A_{\mathrm{tm}_{3}}$. The treatment rate of AIDS females and males who seek treatment is denoted by $\phi$. Treated AIDS patients have a constant disease-induced mortality rate $\tau \nu>0$, with $\tau(0<\tau<1)$, a factor which models the reduced-mortality rate of the treated AIDS individuals in comparison with the untreated AIDS individuals. The model system (22) assumes that the treated AIDS patients $\left(A_{\mathrm{tf}}, A_{\mathrm{tm}_{1}}, A_{\mathrm{tm}_{2}}, A_{\mathrm{tm}_{3}}\right)$ are capable of sexually interacting with the rest of the community spreading the disease with no change in behaviour. The relative infectiousness of the treated AIDS patients is modelled by the parameter $\theta=\epsilon \psi(0 \leq$ $\theta \leq 1$ ) which is a product of drug efficacy $(\epsilon)$ and proportion of the treated AIDS patients who indulge in risk sexual behaviour $(\psi)$. The value $\epsilon=0$ means that the drug is completely effective and $\epsilon=1$ means the drug is useless in stopping HIV transmission. Considering the above explanation, the rates of infection for different classes are given as follows, female susceptibles acquire infection at a time-dependent rate

$$
\lambda_{\mathrm{f}}=\frac{\beta_{\mathrm{m}} c_{\mathrm{f}}\left\{I_{\mathrm{m}_{1}}(t)+\eta I_{\mathrm{m}_{3}}(t)+\theta\left(A_{\mathrm{tm}_{1}}(t)+A_{\mathrm{tm}_{3}}(t)\right)\right\}}{N_{\mathrm{m}_{1}}(t)}
$$

heterosexual male susceptibles acquire infection at a time-dependent rate

$$
\lambda_{m_{1}}=\frac{\beta_{\mathrm{f}} c_{\mathrm{m}_{1}}\left\{I_{\mathrm{f}}(t)+\theta A_{\mathrm{tf}}(t)\right\}}{N_{\mathrm{f}}(t)},
$$

homosexual male population acquire infection at a time-dependent rate

$$
\lambda_{\mathrm{m}_{2}}=\frac{\beta_{\mathrm{h}} c_{\mathrm{m}_{2}}\left\{I_{\mathrm{m}_{2}}(t)+\theta A_{\mathrm{tm}_{2}}(t)\right\}}{N_{\mathrm{m}_{2}}(t)}
$$

The model flow diagram is given in Figure 5. 

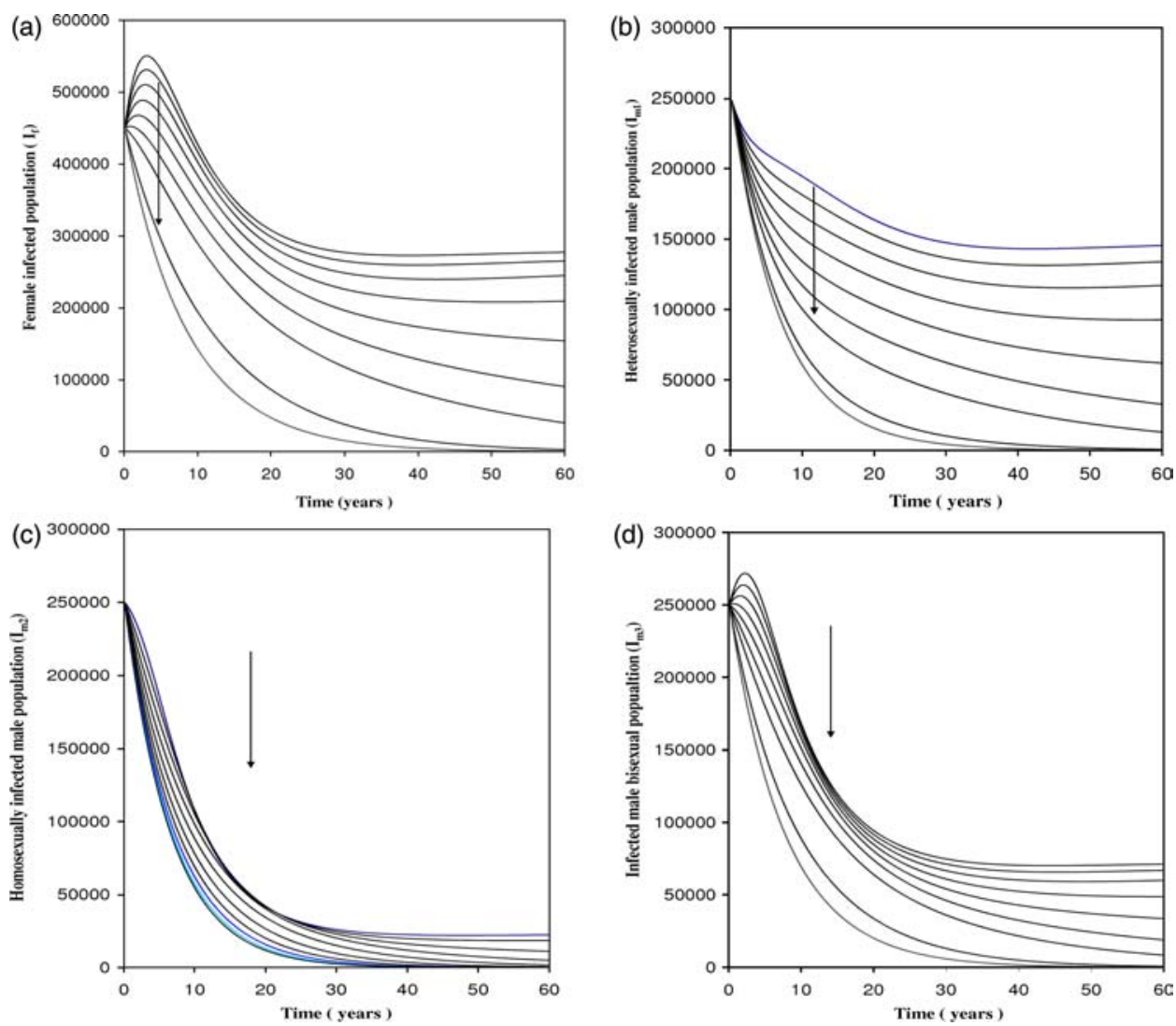

Figure 4. Simulation of model system (4) showing plots of infected female $\left(I_{\mathrm{f}}\right)$, heterosexually infected male $\left(I_{\mathrm{m}_{1}}\right)$, homosexually infected male $\left(I_{\mathrm{m}_{2}}\right)$ and infected male bisexual population $\left(I_{\mathrm{m}_{3}}\right)$ with compliance $\left(\omega_{\mathrm{m}}=\omega_{\mathrm{f}}\right)$ increasing from 0.1 to 1 in step 0.1 when only male condom is in use. Parameters used are from Table 1. The arrow in the figures illustrates the direction of increase in compliance, with assumed initial conditions $S_{\mathrm{f}}=500,000, I_{\mathrm{f}}=450,000, A_{\mathrm{f}}=250,000$, $S_{\mathrm{m}_{1}}=400,000, \quad S_{\mathrm{m}_{2}}=380,000, \quad I_{\mathrm{m}_{1}}=250,000, \quad I_{\mathrm{m}_{2}}=250,000, \quad I_{\mathrm{m}_{3}}=250,000 \quad$ and $A_{\mathrm{m}}=250,000$. The graphs shows that, an increase in compliance in the use of male and female condoms reduces the population of infectives, thus reducing the spread of HIV/AIDS.

Putting the formulations and the assumptions together gives the following system of differential equations,

$$
\begin{gathered}
d \frac{S_{\mathrm{f}}}{\mathrm{d} t}=\pi_{0} \Lambda-\lambda_{\mathrm{f}} S_{\mathrm{f}}(t)-(\alpha+\mu) S_{\mathrm{f}}(t), \\
d \frac{I_{\mathrm{f}}}{\mathrm{d} t}=\lambda_{\mathrm{f}} S_{\mathrm{f}}(t)-(\gamma+\alpha+\mu) I_{\mathrm{f}}(t), \\
d \frac{A_{\mathrm{f}}}{\mathrm{d} t}=\gamma I_{\mathrm{f}}(t)-(\nu+\phi+\mu) A_{\mathrm{f}}(t), \\
d \frac{A_{\mathrm{tf}}}{\mathrm{d} t}=\phi A_{\mathrm{f}}(t)-(\tau \nu+\mu) A_{\mathrm{tf}}(t),
\end{gathered}
$$




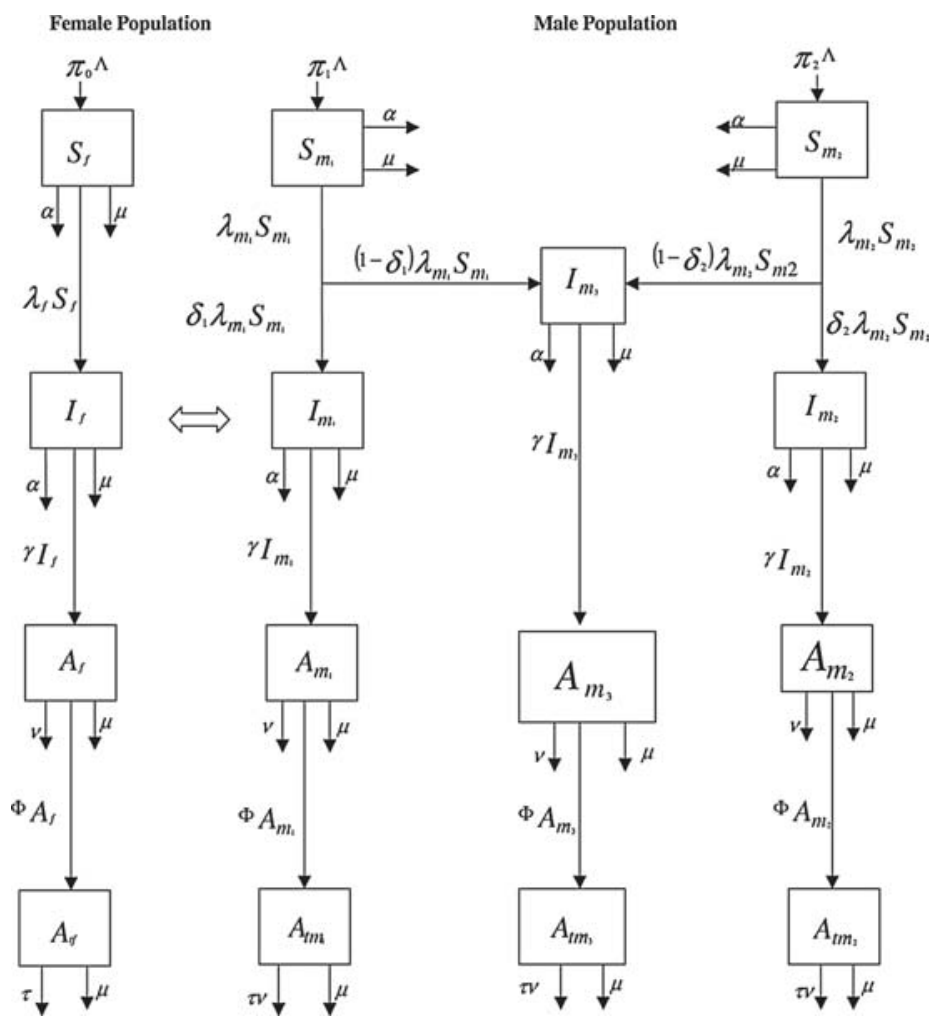

Figure 5. Model flow diagram.

$$
\begin{gathered}
d \frac{S_{\mathrm{m}_{1}}}{\mathrm{~d} t}=\pi_{1} \Lambda-\lambda_{\mathrm{m}_{1}} S_{\mathrm{m}_{1}}(t)-(\alpha+\mu) S_{\mathrm{m}_{1}}(t) \\
d \frac{S_{\mathrm{m}_{2}}}{\mathrm{~d} t}=\pi_{2} \Lambda-\lambda_{\mathrm{m}_{2}} S_{\mathrm{m}_{2}}(t)-(\alpha+\mu) S_{\mathrm{m}_{2}}(t) \\
d \frac{I_{\mathrm{m}_{1}}}{\mathrm{~d} t}=\delta_{1} \lambda_{\mathrm{m}_{1}} S_{\mathrm{m}_{1}}(t)-(\gamma+\alpha+\mu) I_{\mathrm{m}_{1}}(t) \\
d \frac{I_{\mathrm{m}_{2}}}{\mathrm{~d} t}=\delta_{2} \lambda_{\mathrm{m}_{2}} S_{\mathrm{m}_{2}}(t)-(\gamma+\alpha+\mu) I_{\mathrm{m}_{2}}(t) \\
d t=\left(1-I_{1}\right) \lambda_{\mathrm{m}_{1}} S_{\mathrm{m}_{1}}(t)+\left(1-\delta_{2}\right) \lambda_{\mathrm{m}_{2}} S_{\mathrm{m}_{2}}(t)-(\gamma+\alpha+\mu) I_{\mathrm{m}_{3}}(t), \\
d \frac{A_{\mathrm{m}_{1}}}{\mathrm{~d} t}=\gamma I_{\mathrm{m}_{1}}(t)-(\nu+\phi+\mu) A_{\mathrm{m}_{1}}(t) \\
d \frac{A_{\mathrm{m}_{2}}}{\mathrm{~d} t}=\gamma I_{\mathrm{m}_{2}}(t)-(\nu+\phi+\mu) A_{\mathrm{m}_{2}}(t)
\end{gathered}
$$




$$
\begin{gathered}
d \frac{A_{\mathrm{m}_{3}}}{\mathrm{~d} t}=\gamma I_{\mathrm{m}_{3}}(t)-(\nu+\phi+\mu) A_{\mathrm{m}_{3}}(t), \\
d \frac{A_{\mathrm{tm}_{1}}}{\mathrm{~d} t}=\phi A_{\mathrm{m}_{1}}(t)-(\tau \nu+\mu) A_{\mathrm{tm}_{1}}(t) \\
d \frac{A_{\mathrm{tm}_{2}}}{\mathrm{~d} t}=\phi A_{\mathrm{m}_{2}}(t)-(\tau \nu+\mu) A_{\mathrm{tm}_{2}}(t), \\
d \frac{A_{\mathrm{tm}_{3}}}{\mathrm{~d} t}=\phi A_{\mathrm{m}_{3}}(t)-(\tau \nu+\mu) A_{\mathrm{tm}_{3}}(t)
\end{gathered}
$$

Next, we consider the equilibria, reproductive numbers and stabilities of the model.

\subsection{DFE and reproductive number}

Model system (22) has a DFE given by,

$$
\begin{aligned}
\mathcal{E}^{0} & =\left(S_{\mathrm{f}}^{0}, S_{\mathrm{m}_{1}}^{0}, S_{\mathrm{m}_{2}}^{0}, I_{\mathrm{f}}^{0}, I_{\mathrm{m}_{1}}^{0}, I_{\mathrm{m}_{2}}^{0}, I_{\mathrm{m}_{3}}^{0}, A_{\mathrm{f}}^{0}, A_{\mathrm{tf}}^{0}, A_{\mathrm{m}_{1}}^{0}, A_{\mathrm{m}_{2}}^{0}, A_{\mathrm{m}_{3}}^{0}, A_{\mathrm{tm}_{1}}^{0}, A_{\mathrm{tm}_{2}}^{0}, A_{\mathrm{tm}_{3}}^{0}\right) \\
& =\left(\frac{\pi_{0} \Lambda}{\alpha+\mu}, \frac{\pi_{1} \Lambda}{\alpha+\mu}, \frac{\pi_{2} \Lambda}{\alpha+\mu}, 0,0,0,0,0,0,0,0,0,0,0,0\right) .
\end{aligned}
$$

The linear stability of $\mathcal{E}^{0}$ is governed by the reproductive number $\mathcal{R}_{0}[1,6,9,12]$. Using the notation in [20] on the model system (22), the matrices $\mathbf{F}$ and $\mathbf{V}$, for the new infection terms and the remaining transfer terms are, respectively, given by

$$
\mathbf{F}=\left(\begin{array}{cccccccccccc}
0 & 0 & 0 & k_{1} & 0 & \eta k_{1} & 0 & 0 & 0 & \theta k_{1} & 0 & \theta k_{1} \\
0 & 0 & 0 & 0 & 0 & 0 & 0 & 0 & 0 & 0 & 0 & 0 \\
0 & 0 & 0 & 0 & 0 & 0 & 0 & 0 & 0 & 0 & 0 & 0 \\
k_{2} & 0 & \theta k_{2} & 0 & 0 & 0 & 0 & 0 & 0 & 0 & 0 & 0 \\
0 & 0 & 0 & 0 & k_{3} & 0 & 0 & 0 & 0 & 0 & \theta k_{3} & 0 \\
k_{4} & 0 & \theta k_{4} & 0 & k_{5} & 0 & 0 & 0 & 0 & 0 & \theta k_{5} & 0 \\
0 & 0 & 0 & 0 & 0 & 0 & 0 & 0 & 0 & 0 & 0 & 0 \\
0 & 0 & 0 & 0 & 0 & 0 & 0 & 0 & 0 & 0 & 0 & 0 \\
0 & 0 & 0 & 0 & 0 & 0 & 0 & 0 & 0 & 0 & 0 & 0 \\
0 & 0 & 0 & 0 & 0 & 0 & 0 & 0 & 0 & 0 & 0 & 0 \\
0 & 0 & 0 & 0 & 0 & 0 & 0 & 0 & 0 & 0 & 0 & 0 \\
0 & 0 & 0 & 0 & 0 & 0 & 0 & 0 & 0 & 0 & 0 & 0
\end{array}\right) .
$$

Here

$$
\begin{aligned}
& k_{1}=\frac{\pi_{0} \beta_{\mathrm{m}} c_{\mathrm{f}}}{\pi_{1}}, \quad k_{2}=\frac{\delta_{1} \pi_{1} \beta_{\mathrm{f}} c_{m 1}}{\pi_{0}}, \quad k_{3}=\delta_{2} \beta_{\mathrm{h}} c_{m 2}, \\
& k_{4}=\frac{\left(1-\delta_{1}\right) \pi_{1} \beta_{\mathrm{f}} c_{m 1}}{\pi_{0}}, \quad k_{5}=\left(1-\delta_{2}\right) \theta \beta_{\mathrm{h}} c_{m 2}
\end{aligned}
$$


and

$$
\mathbf{V}=\left(\begin{array}{cccccccccccc}
b & 0 & 0 & 0 & 0 & 0 & 0 & 0 & 0 & 0 & 0 & 0 \\
-\gamma & a & 0 & 0 & 0 & 0 & 0 & 0 & 0 & 0 & 0 & 0 \\
0 & -\phi & d & 0 & 0 & 0 & 0 & 0 & 0 & 0 & 0 & 0 \\
0 & 0 & 0 & b & 0 & 0 & 0 & 0 & 0 & 0 & 0 & 0 \\
0 & 0 & 0 & 0 & b & 0 & 0 & 0 & 0 & 0 & 0 & 0 \\
0 & 0 & 0 & 0 & 0 & b & 0 & 0 & 0 & 0 & 0 & 0 \\
0 & 0 & 0 & -\gamma & 0 & 0 & a & 0 & 0 & 0 & 0 & 0 \\
0 & 0 & 0 & 0 & -\gamma & 0 & 0 & a & 0 & 0 & 0 & 0 \\
0 & 0 & 0 & 0 & 0 & -\gamma & 0 & 0 & a & 0 & 0 & 0 \\
0 & 0 & 0 & 0 & 0 & 0 & -\phi & 0 & 0 & d & 0 & 0 \\
0 & 0 & 0 & 0 & 0 & 0 & 0 & -\phi & 0 & 0 & d & 0 \\
0 & 0 & 0 & 0 & 0 & 0 & 0 & 0 & -\phi & 0 & 0 & d
\end{array}\right) .
$$

Here $a=\phi+\nu+\mu, b=\alpha+\gamma+\mu$ and $d=\tau \nu+\mu$. It follows that the treatmentinduced reproductive number $\left(\mathcal{R}_{\mathrm{t}}\right)$ for the model system $(22)$ is given by

$$
\mathcal{R}_{\mathrm{t}}=\rho\left(\mathbf{F V}^{-1}\right)=\max \left\{\mathcal{R}_{\mathrm{t}_{1}}, \mathcal{R}_{\mathrm{t}_{2}}\right\}
$$

where

$$
\begin{aligned}
\mathcal{R}_{\mathrm{t}_{1}} & =\frac{\delta_{2} \beta_{\mathrm{h}} c_{m 2}}{\alpha+\gamma+\mu} f_{1}(\theta, \phi)=\mathcal{R}_{01} f_{1}(\theta, \phi) \\
& =\text { with } f_{1}(\theta, \phi)\left\{1+\frac{\gamma \theta \phi}{(\phi+\mu+\nu)(\tau \nu+\mu)}\right\}
\end{aligned}
$$

and

$$
\mathcal{R}_{\mathrm{t}_{2}}=f_{2}(\theta, \phi) \sqrt{\frac{\beta_{\mathrm{f}} \beta_{\mathrm{m}} c_{\mathrm{f}} c_{\mathrm{m}_{1}}}{(\alpha+\gamma+\mu)^{2}}\left[\delta_{1}+\eta\left(1-\delta_{1}\right)\right]}=\mathcal{R}_{02} f_{2}(\theta, \phi)
$$

with

$f_{2}(\theta, \phi)=\sqrt{\left\{1+\frac{\gamma \theta \phi}{(\phi+\mu+\nu)(\tau \nu+\mu)}\right\}\left\{1+\frac{\gamma \theta \phi}{(\phi+\mu+\nu)(\tau \nu+\mu)\left[\delta_{1}+\eta\left(1-\delta_{1}\right)\right]}\right\}}$.

The parameters $f_{1}(\theta, \phi)$ and $f_{2}(\theta, \phi)$ are a measure of the effectiveness of treatment in a community. Using Theorem 2 in [20], the following result is established.

LEMMA 2. The DFE of model system (22) is locally asymptotically stable (LAS) if $\mathcal{R}_{\mathrm{t}}<1$ and unstable if $\mathcal{R}_{\mathrm{t}}>1$. 
It is also important to note that $\left(f_{1}(\theta, \phi), f_{2}(\theta, \phi)\right)>1$, which shows that the treatment increases the average number of new infections generated by a single infected individual in a completely susceptible population thus may enlarge the epidemic. We analyse $\mathcal{R}_{\mathrm{t}_{1}}$ for the following cases.

Case 1. When $\theta=0$

$$
\mathcal{R}_{\mathrm{t}_{1}}=\mathcal{R}_{01}=\frac{\delta_{2} \beta_{\mathrm{h}} c_{m 2}}{\alpha+\gamma+\mu}, \quad \mathcal{R}_{\mathrm{t}_{2}}=\mathcal{R}_{02}=\sqrt{\frac{\beta_{\mathrm{f}} \beta_{\mathrm{m}} c_{\mathrm{f}} c_{\mathrm{m}_{1}}}{(\alpha+\gamma+\mu)^{2}}\left[\delta_{1}+\eta\left(1-\delta_{1}\right)\right]},
$$

where $\mathcal{R}_{\mathrm{t}_{1}}$ is the treatment-induced reproductive number for homosexuals and $\mathcal{R}_{\mathrm{t}_{2}}$ is the treatment-induced reproductive number for heterosexuals and bisexuals. Note that $\mathcal{R}_{01}$ and $\mathcal{R}_{02}$ are the basic reproductive numbers when there are no HIV/AIDS intervention strategies in a community, for homosexuals and for both the heterosexuals and bisexuals, respectively. We note that the factor multiplying the basic reproductive number $\left(\mathcal{R}_{01}\right.$ and $\left.\mathcal{R}_{02}\right)$ is equal to unity $\left(f_{1}(\theta, \phi)\right.$ and $\left.f_{2}(\theta, \phi)=1\right)$, suggesting that either the treatment drug is $100 \%$ effective $(\epsilon=0)$ and /or treated patients are not indulging in risk sexual activities $(\psi=0)$. Thus the intervention has no effect on the basic reproductive number but helps the community by lengthening the lives of AIDS patients, reducing morbidity/mortality and reducing the number of orphans in affected communities.

Case 2. When $0<\theta \leq 1$

$$
\mathcal{R}_{\mathrm{t}_{1}}=\frac{\delta_{2} \beta_{\mathrm{h}} c_{m 2}}{\alpha+\gamma+\mu} f_{1}(\theta, \phi)=\mathcal{R}_{01} f_{1}(\theta, \phi)
$$

where

$$
f_{1}(\theta, \phi)=\left\{1+\frac{\gamma \theta \phi}{(\phi+\mu+\nu)(\tau \nu+\mu)}\right\}
$$

and

$$
\mathcal{R}_{\mathrm{t}_{2}}=f_{2}(\theta, \phi) \sqrt{\frac{\beta_{\mathrm{f}} \beta_{\mathrm{m}} c_{\mathrm{f}} c_{\mathrm{m}_{1}}}{(\alpha+\gamma+\mu)^{2}}\left[\delta_{1}+\eta\left(1-\delta_{1}\right)\right]}=\mathcal{R}_{02} f_{2}(\theta, \phi)
$$

where

$f_{2}(\theta, \phi)=\sqrt{\left\{1+\frac{\gamma \theta \phi}{(\phi+\mu+\nu)(\tau \nu+\mu)}\right\}\left\{1+\frac{\gamma \theta \phi}{(\phi+\mu+\nu)(\tau \nu+\mu)\left[\delta_{1}+\eta\left(1-\delta_{1}\right)\right]}\right\}}$

We note that the factor multiplying the basic reproductive number $\left(\mathcal{R}_{01}\right.$ and $\left.\mathcal{R}_{02}\right)$ is greater than unity $\left(f_{1}(\theta, \phi)\right.$ and $\left.f_{2}(\theta, \phi)>1\right)$ and this is when the treatment drug is not $100 \%$ effective $(0<\epsilon \leq 1)$ and when treated AIDS patients indulge in risk sexual behaviour $(0<\psi \leq 1)$. This is a realistic scenario which suggests that the treatment of AIDS patients enlarges the epidemic. Clinical trials with highly active antiretroviral therapy (HAART) have shown that treatment reduces the plasma HIV-RNA to undetectable levels, 
and thus due to the reduction in seminal viral load, heterosexual transmission of HIV (infectiousness) may be reduced [11]. However, the main function of current HAART is to increase the life expectancy of infected individuals and over time this causes the pool of potential transmitters of infection to grow, thus the two factors, decreased infectivity but increased duration of infectiousness, have opposing effects on transmission [3].

\subsection{Effects of condom use in settings with the treatment}

Introducing condom use to model system (22) gives the condom and treatment-induced reproductive number as

$$
\mathcal{R}_{\mathrm{ct}}=\max \left\{\sigma_{\mathrm{m}} \mathcal{R}_{\mathrm{t}_{1}}, \sigma_{\mathrm{fm}} \mathcal{R}_{\mathrm{t}_{2}}\right\}
$$

and we note here that $\mathcal{R}_{\mathrm{ct}}<\mathcal{R}_{\mathrm{t}}$ since $\left(\sigma_{\mathrm{m}}, \sigma_{\mathrm{fm}}\right)<1$. Note that here we consider the use of male condoms only (modelled by $\sigma_{\mathrm{m}}$ ) by the homosexuals and the use of male and female condoms by the bisexuals and heterosexuals (modelled by $\sigma_{\mathrm{fm}}$ ). Thus we conclude that the use of condoms in settings with the treatment will help significantly in slowing the epidemic.

\subsection{Global stability of the DFE}

Lemma 2 implies that HIV/AIDS can be eliminated from the community (when $\mathcal{R}_{\mathrm{t}}<1$ ) if the initial sizes of the sub-populations of the model are in the basin of attraction of the DFE. To ensure that the elimination of the virus is independent of the initial sizes of the subpopulations, it is necessary to show that the DFE is globally asymptotically stable (GAS). Here, we examine the global stability of model system (22) at the DFE when a considerable number of infectives are introduced in a totally susceptible population using the method in [9]. Let $X=\left(S_{\mathrm{f}}, S_{\mathrm{m}_{1}}, S_{\mathrm{m}_{2}}\right)$ and $Z=\left(I_{\mathrm{f}}, I_{\mathrm{m}_{1}}, I_{\mathrm{m}_{2}}, I_{\mathrm{m}_{3}}, A_{\mathrm{f}}, A_{\mathrm{t}_{\mathrm{f}}}, A_{\mathrm{m}_{1}}, A_{\mathrm{m}_{2}}, A_{\mathrm{m}_{3}}, A_{\mathrm{tm}_{1}}, A_{\mathrm{tm}_{2}}, A_{\mathrm{tm}_{3}}\right)$, thus $\mathbf{F}(X, 0)$ and $\hat{\mathbf{G}}(X, Z)$ are given as follows:

$$
\mathbf{F}(X, 0)=\left(\begin{array}{c}
\pi_{0} \Lambda-(\alpha+\mu) S_{\mathrm{f}} \\
\pi_{1} \Lambda-(\alpha+\mu) S_{\mathrm{m}_{1}} \\
\pi_{2} \Lambda-(\alpha+\mu) S_{\mathrm{m}_{2}}
\end{array}\right) \text { and } \hat{\mathbf{G}}(X, Z)=\left(\begin{array}{c}
\hat{\mathbf{G}}_{1}(X, Z) \\
\hat{\mathbf{G}}_{2}(X, Z) \\
\hat{\mathbf{G}}_{3}(X, Z) \\
\hat{\mathbf{G}}_{4}(X, Z)
\end{array}\right) \geq 0
$$

with

$$
\begin{aligned}
\hat{\mathbf{G}}_{1}(X, Z)= & \beta_{\mathrm{m}} c_{\mathrm{f}}\left(I_{\mathrm{m}_{1}}+\eta I_{\mathrm{m}_{3}}+\theta\left(A_{\mathrm{tm}_{1}}+A_{\mathrm{tm}_{3}}\right)\right)\left(\frac{\pi_{0}}{\pi_{1}}-\frac{S_{\mathrm{f}}}{S_{\mathrm{m}_{1}}+I_{\mathrm{m}_{1}}+I_{\mathrm{m}_{3}}+A_{\mathrm{tm}_{1}}+A_{\mathrm{tm}_{3}}}\right), \\
\hat{\mathbf{G}}_{2}(X, Z)= & \delta_{1} \beta_{\mathrm{f}} c_{\mathrm{m}_{1}}\left(I_{\mathrm{f}}+\theta A_{\mathrm{tf}}\right)\left(\frac{\pi_{1}}{\pi_{0}}-\frac{S_{\mathrm{m}_{1}}}{S_{\mathrm{f}}+I_{\mathrm{f}}+A_{\mathrm{t}_{\mathrm{f}}}}\right), \\
\hat{\mathbf{G}}_{3}(X, Z)= & \delta_{2} \beta_{\mathrm{h}} c_{\mathrm{m}_{2}}\left(I_{\mathrm{m}_{2}}+\theta A_{\mathrm{tm}_{2}}\right)\left(1-\frac{S_{\mathrm{m}_{2}}}{S_{\mathrm{m}_{2}}+I_{\mathrm{m}_{2}}+A_{\mathrm{tm}_{2}}}\right), \\
\hat{\mathbf{G}}_{4}(X, Z)= & \left(1-\delta_{1}\right) \beta_{\mathrm{f}} c_{\mathrm{m}_{1}}\left(I_{\mathrm{f}}+A_{\mathrm{tf}}\right)\left(\frac{\pi_{1}}{\pi_{0}}-\frac{S_{\mathrm{m}_{1}}}{S_{\mathrm{f}}+I_{\mathrm{f}}+A_{\mathrm{tf}}}\right) \\
& +\left(1-\delta_{2}\right) \beta_{\mathrm{h}} c_{\mathrm{m}_{2}}\left(I_{\mathrm{m}_{2}}+A_{\mathrm{tm}_{2}}\right)\left(1-\frac{S_{\mathrm{m}_{2}}}{S_{\mathrm{m}_{2}}+I_{\mathrm{m}_{2}+} A_{\mathrm{tm}_{2}}}\right) .
\end{aligned}
$$

Since $\hat{\mathbf{G}}(X, Z) \geq 0 \mathcal{E}^{0}$ is GAS. We summarize the result in Lemma 3. 
Lemma 3. If $\mathcal{R}_{\mathrm{t}}<1$, the DFE of model system (22) is GAS and unstable if $\mathcal{R}_{\mathrm{t}}>1$.

\subsection{Endemic equilibrium (EE)}

Model system (22) has an EE given by,

$$
\mathcal{E}^{*}=\left\{\begin{array}{l}
S_{\mathrm{f}}^{*}=\frac{\pi_{0} \Lambda}{\alpha+\mu+\lambda_{\mathrm{f}}^{*}}, S_{\mathrm{m}_{1}}^{*}=\frac{\pi_{1} \Lambda}{\alpha+\mu+\lambda_{\mathrm{m}_{1}}^{*}}, S_{\mathrm{m}_{2}}^{*}=\frac{\pi_{2} \Lambda}{\alpha+\mu+\lambda_{\mathrm{m}_{2}}^{*}}, I_{\mathrm{f}}^{*}=\frac{\pi_{0} \Lambda \lambda_{\mathrm{f}}^{*}}{(\alpha+\gamma+\mu)\left(\alpha+\mu+\lambda_{\mathrm{f}}^{*}\right)}, \\
I_{\mathrm{m}_{1}}^{*}=\frac{\delta_{1} \pi_{1} \Lambda \lambda_{\mathrm{m}_{1}}^{*}}{(\alpha+\gamma+\mu)\left(\alpha+\mu+\lambda_{\mathrm{m}_{1}}^{*}\right)}, I_{\mathrm{m}_{2}}^{*}=\frac{\delta_{2} \pi_{2} \Lambda \lambda_{\mathrm{m}_{2}}^{*}}{(\alpha+\gamma+\mu)\left(\alpha+\mu+\lambda_{\mathrm{m}_{2}}^{*}\right)}, \\
I_{\mathrm{m}_{3}}^{*}=\frac{\Lambda}{(\alpha+\gamma+\mu)}\left(\frac{\left(1-\delta_{1}\right) \pi_{1} \lambda_{\mathrm{m}_{1}}^{*}}{\alpha+\mu+\lambda_{\mathrm{m}_{1}}^{*}}+\frac{\left(1-\delta_{2}\right) \pi_{2} \lambda_{\mathrm{m}_{2}}^{*}}{\alpha+\mu+\lambda_{\mathrm{m}_{2}}^{*}}\right), A_{\mathrm{f}}^{*}=\frac{\pi_{0} \Lambda \gamma \lambda_{\mathrm{f}}^{*}}{(\mu+\nu)(\alpha+\gamma+\mu)\left(\alpha+\phi+\mu+\lambda_{\mathrm{f}}^{*}\right)}, \\
A_{\mathrm{m}_{1}}^{*}=\frac{\delta_{1} \pi_{1} \Lambda \lambda_{\mathrm{m}_{1}}^{*}}{(\alpha+\gamma+\mu)\left(\alpha+\mu+\lambda_{\mathrm{m}_{1}}^{*}\right)} \frac{\gamma}{(\nu+\phi+\mu)}, A_{\mathrm{m}_{2}}^{*}=\frac{\delta_{2} \pi_{2} \Lambda \lambda_{\mathrm{m}_{2}}^{*}}{(\alpha+\gamma+\mu)\left(\alpha+\mu+\lambda_{\mathrm{m}_{2}}^{*}\right)} \frac{\gamma}{(\nu+\phi+\mu)}, \\
A_{\mathrm{m}_{3}}^{*}=\frac{\Lambda}{(\alpha+\gamma+\mu)}\left(\frac{\left(1-\delta_{1}\right) \pi_{1} \lambda_{\mathrm{m}_{1}}^{*}}{\alpha+\mu+\lambda_{\mathrm{m}_{1}}^{*}}+\frac{\left(1-\delta_{2}\right) \pi_{2} \lambda_{\mathrm{m}_{2}}^{*}}{\alpha+\mu+\lambda_{\mathrm{m}_{2}}^{*}}\right) \frac{\gamma}{(\nu+\phi+\mu)}, \\
A_{\mathrm{tf}}^{*}=\frac{\pi_{0} \Lambda \gamma \lambda_{\mathrm{f}}^{*}}{(\mu+\nu)(\alpha+\gamma+\mu)\left(\alpha+\phi+\mu+\lambda_{\mathrm{f}}^{*}\right)} \frac{\phi}{(\tau \nu+\mu)}, \\
A_{\mathrm{tm}_{1}}^{*}=\frac{\delta_{1} \pi_{1} \Lambda \lambda_{\mathrm{m}_{1}}^{*}}{(\alpha+\gamma+\mu)\left(\alpha+\mu+\lambda_{\left.\mathrm{m}_{1}\right)}^{*}\right)} \frac{\gamma}{(\nu+\phi+\mu)} \frac{\phi}{(\tau \nu+\mu)}, \\
A_{\mathrm{tm}_{2}}^{*}=\frac{\delta_{2} \pi_{2} \Lambda \lambda_{\mathrm{m}_{2}}^{*}}{(\alpha+\gamma+\mu)\left(\alpha+\mu+\lambda_{\mathrm{m}_{2}}^{*}\right)} \frac{\gamma}{(\nu+\phi+\mu)} \frac{\phi}{(\tau \nu+\mu)}, \\
A_{\mathrm{tm}_{3}}^{*}=\frac{\Lambda}{(\alpha+\gamma+\mu)}\left(\frac{\left(1-\delta_{1}\right) \pi_{1} \lambda_{\mathrm{m}_{1}}^{*}}{\alpha+\mu+\lambda_{\mathrm{m}_{1}}^{*}}+\frac{\left(1-\delta_{2}\right) \pi_{2} \lambda_{\mathrm{m}_{2}}^{*}}{\alpha+\mu+\lambda_{\mathrm{m}_{2}}^{*}}\right) \frac{\gamma}{(\nu+\phi+\mu)} \frac{\phi}{(\tau \nu+\mu)},
\end{array}\right.
$$

where

$$
\begin{aligned}
\lambda_{\mathrm{f}}^{*} & =\frac{\beta_{\mathrm{m}} c_{\mathrm{f}}\left\{I_{\mathrm{m}_{1}}^{*}+\eta I_{\mathrm{m}_{3}}^{*}+\theta\left(A_{\mathrm{tm}_{1}}^{*}+A_{\mathrm{tm}_{3}}^{*}\right)\right\}}{N_{\mathrm{m}_{1}}^{*}}, \lambda_{\mathrm{m}_{1}}^{*}=\beta_{\mathrm{f}} c_{\mathrm{m}_{1}} \frac{\left(I_{\mathrm{f}}^{*}+\theta A_{\mathrm{tf}}^{*}\right)}{N_{\mathrm{f}}^{*}} \text { and } \\
\lambda_{\mathrm{m}_{2}}^{*} & =\beta_{\mathrm{h}} c_{\mathrm{m}_{2}} \frac{\left(I_{\mathrm{m}_{2}}^{*}+\theta A_{\mathrm{tm}_{2}}^{*}\right)}{N_{\mathrm{m}_{2}}^{*}} .
\end{aligned}
$$

We now apply the centre manifold theory [7] as described in [8] (Theorem 4.1) to establish the local asymptotic stability of the EE. We make the following change of variables, $\quad S_{\mathrm{f}}=x_{1}, I_{\mathrm{f}}=x_{2}, A_{\mathrm{f}}=x_{3}, A_{\mathrm{tf}}=x_{4}, S_{\mathrm{m}_{1}}=x_{5}, I_{\mathrm{m}_{1}}=x_{6}, I_{\mathrm{m}_{3}}=x_{7}=x_{9}, A_{\mathrm{tm}_{1}}=$ $x_{10}, A_{\mathrm{tm}_{3}}=x_{11}, S_{\mathrm{m}_{2}}=x_{12}, I_{\mathrm{m}_{2}}=x_{13}, A_{\mathrm{m}_{2}}=x_{14}$ and $A_{\mathrm{tm}_{2}}=x_{15}$ so that $N=\sum_{n=1}^{15} x_{n}$, $N_{\mathrm{f}}=x_{1}+x_{2}+x_{4}, N_{\mathrm{m}_{1}}=x_{5}+x_{6}+x_{7}+x_{10}+x_{11}, N_{\mathrm{m}_{2}}=x_{12}+x_{13}+x_{15}$. Using the vector notation $X=\left(x_{1}, x_{2}, x_{3}, x_{4}, x_{5}, x_{6}, x_{7}, x_{8}, x_{9}, x_{10}, x_{11}, x_{12}, x_{13}, x_{14}, x_{15}\right)^{\mathrm{T}}$, model system (22) can be written in the form $\mathrm{d} X / \mathrm{d} t=\mathbf{F}=\left(f_{1}, f_{2}, f_{3}, f_{4}, f_{5}, f_{6}, f_{7}, f_{8}, f_{9}, f_{10}\right.$, $\left.f_{11}, f_{12}, f_{13}, f_{14}, f_{15}\right)^{\mathrm{T}}$, such that;

$$
\begin{aligned}
\lambda_{\mathrm{f}} & =\frac{\beta_{\mathrm{m}} c_{\mathrm{f}}\left\{x_{6}+\eta x_{7}+\theta\left(x_{10}+x_{11}\right)\right\}}{N_{\mathrm{m}_{1}}}, \lambda_{\mathrm{m}_{1}}=\beta_{\mathrm{f}} c_{\mathrm{m}_{1}} \frac{\left(x_{2}+\theta x_{3}\right)}{N_{\mathrm{f}}}, \\
\lambda_{\mathrm{m}_{2}} & =\beta_{\mathrm{h}} c_{\mathrm{m}_{2}} \frac{\left(x_{13}+\theta x_{15}\right)}{N_{\mathrm{m}_{2}}}
\end{aligned}
$$




$$
\begin{gathered}
x_{1}^{\prime}(t)=f_{1}=\pi_{0} \Lambda-\lambda_{\mathrm{f}} x_{1}(t)-(\alpha+\mu) x_{1}(t), \\
x_{2}^{\prime}(t)=f_{2}=\lambda_{\mathrm{f}} x_{1}(t)-(\gamma+\alpha+\mu) x_{2}(t), \\
x_{3}^{\prime}(t)=f_{3}=\gamma x_{2}(t)-(\nu+\phi+\mu) x_{3}(t), \\
x_{4}^{\prime}(t)=f_{4}=\phi x_{3}(t)-(\tau \nu+\mu) x_{4}(t), \\
x_{5}^{\prime}(t)=f_{5}=\pi_{1} \Lambda-\lambda_{\mathrm{m}_{1}} x_{5}(t)-(\alpha+\mu) x_{5}(t), \\
x_{6}^{\prime}(t)=f_{6}=\delta_{1} \lambda_{\mathrm{m}_{1}} x_{5}(t)-(\gamma+\alpha+\mu) x_{6}(t), \\
x_{7}^{\prime}(t)=f_{7}=\left(1-\delta_{1}\right) \lambda_{\mathrm{m}_{1}} x_{5}(t)+\left(1-\delta_{2}\right) \lambda_{\mathrm{m}_{2}} x_{12}(t)-(\gamma+\alpha+\mu) x_{7}(t), \\
x_{8}^{\prime}(t)=f_{8}=\gamma x_{6}(t)-(\nu+\phi+\mu) x_{8}(t), \\
x_{9}^{\prime}(t)=f_{9}=\gamma x_{7}(t)-(\nu+\phi+\mu) x_{9}(t), \\
x_{10}^{\prime}(t)=f_{10}=\phi x_{8}(t)-(\tau \nu+\mu) x_{10}(t), \\
x_{11}^{\prime}(t)=f_{11}=\phi x_{9}(t)-(\tau \nu+\mu) x_{11}(t), \\
x_{12}^{\prime}(t)=f_{12}=\pi_{2} \Lambda-\lambda_{\mathrm{m}_{2}} x_{12}(t)-(\alpha+\mu) x_{12}(t), \\
x_{13}^{\prime}(t)=f_{13}=\delta_{2} \lambda_{\mathrm{m}_{2}} x_{12}(t)-(\gamma+\alpha+\mu) x_{13}(t), \\
x_{14}^{\prime}(t)=f_{14}=\gamma x_{13}(t)-(\nu+\phi+\mu) x_{14}(t), \\
x_{15}^{\prime}(t)=f_{15}=\phi x_{14}(t)-(\tau \nu+\mu) x_{15}(t) .
\end{gathered}
$$

Consider the equations $x_{12}^{\prime}(t), x_{13}^{\prime}(t), x_{14}^{\prime}(t)$ and $x_{15}^{\prime}(t)$ which describe purely homosexuals, that is;

$$
\begin{gathered}
x_{12}^{\prime}(t)=f_{12}=\pi_{2} \Lambda-\lambda_{\mathrm{m}_{2}} x_{12}(t)-(\alpha+\mu) x_{12}(t), \\
x_{13}^{\prime}(t)=f_{13}=\delta_{2} \lambda_{\mathrm{m}_{2}} x_{12}(t)-(\gamma+\alpha+\mu) x_{13}(t), \\
x_{14}^{\prime}(t)=f_{14}=\gamma x_{13}(t)-(\nu+\phi+\mu) x_{14}(t), \\
x_{15}^{\prime}(t)=f_{15}=\phi x_{14}(t)-(\tau \nu+\mu) x_{15}(t) .
\end{gathered}
$$

The Jacobian of the system (30) is given by,

$$
\mathbf{F}=\left(\begin{array}{cccc}
-\alpha-\mu & \beta_{\mathrm{h}} c_{\mathrm{m}_{2}} & 0 & \theta \beta_{\mathrm{h}} c_{\mathrm{m}_{2}} \\
0 & \delta_{2} \beta_{\mathrm{h}} c_{\mathrm{m}_{2}}-(\alpha+\gamma+\mu) & 0 & \delta_{2} \theta \beta_{\mathrm{h}} c_{\mathrm{m}_{2}} \\
0 & \gamma & -\mu-\nu-\phi & 0 \\
0 & 0 & \phi & -\mu-\tau \nu
\end{array}\right) .
$$

From (31) it can be shown that,

$$
\mathcal{R}_{\mathrm{t}}=\frac{\delta_{2} \beta_{\mathrm{h}} c_{m 2}}{\alpha+\gamma+\mu}\left\{1+\frac{\gamma \theta \phi}{(\phi+\mu+\nu)(\tau \nu+\mu)}\right\}
$$


If $\beta_{\mathrm{h}}$ is taken as a bifurcation point and if we consider the case $\mathcal{R}_{\mathrm{t}}=1$ and solving for $\beta_{\mathrm{h}}$ gives,

$$
\beta_{\mathrm{h}}=\beta^{*}=\frac{(\alpha+\gamma+\mu)}{\delta_{2} c_{\mathrm{m}_{2}}} \frac{(\phi+\mu+\nu)(\tau \nu+\mu)}{[\gamma \theta \phi+(\phi+\mu+\nu)(\tau \nu+\mu)]} .
$$

Then the linearized system of the transformed Equation (30) with $\beta_{\mathrm{h}}=\beta^{*}$ has a simple zero eigenvalue. Hence the centre manifold theory [7] can be used to analyse the dynamics of (30) near $\beta^{*}$. It can be shown that the Jacobian (31) at $\beta^{*}=\beta_{\mathrm{h}}$ has a right eigenvector associated with the zero eigenvalue given by $u=\left(u_{12}, u_{13}, u_{14}, u_{15}\right)^{\mathrm{T}}$, where

$$
\begin{gathered}
u_{12}=\frac{\phi \theta \beta^{*} c_{\mathrm{m}_{2}}}{(\alpha+\mu)(\tau \nu+\mu)}\left\{1+\frac{\delta_{2} \beta^{*} c_{\mathrm{m}_{2}}}{(\alpha+\gamma+\mu)-\delta_{2} \beta^{*} c_{\mathrm{m}_{2}}}\right\} u_{14}, \\
u_{13}=\frac{\phi \theta \delta_{2} \beta^{*} c_{\mathrm{m}_{2}}}{(\tau \nu+\mu)\left[(\alpha+\gamma+\mu)-\delta_{2} \beta^{*} c_{\mathrm{m}_{2}}\right]} u_{14}, u_{14}=u_{14}>0, \quad u_{15}=\frac{\phi}{(\tau \nu+\mu)} u_{14} .
\end{gathered}
$$

The left eigenvector of $J\left(\mathcal{E}^{0}\right)$ (31) associated with the zero eigenvalue at $\beta^{*}=\beta_{\mathrm{h}}$ is given by $v=\left(v_{12}, v_{13}, v_{14}, v_{15}\right)^{\mathrm{T}}$, where

$$
\begin{gathered}
v_{12}=0, v_{13}=\frac{\gamma}{(\alpha+\gamma+\mu)-\delta_{2} \beta^{*} c_{\mathrm{m}_{2}}} v_{14}, \quad v_{14}=v_{14}, \\
v_{15}=\frac{\gamma \theta \delta_{2} \beta^{*} c_{\mathrm{m}_{2}}}{(\tau \nu+\mu)\left[(\alpha+\gamma+\mu)-\delta_{2} \beta^{*} c_{\mathrm{m}_{2}}\right]} v_{14} .
\end{gathered}
$$

In order to establish the conditions for the existence of a bifurcation, we use Theorem 1 proven in [8].

THEOREM 1. Consider the following general system of ordinary differential equations with a parameter $\phi$,

$$
\frac{\mathrm{d} x}{\mathrm{~d} t}=f(x, \phi), f: \mathbb{R}^{n} \times \mathbb{R} \rightarrow \text { and } f \in \mathbb{C}^{2}\left(\mathbb{R}^{n} \times \mathbb{R}\right),
$$

where 0 is an equilibrium of the system that is $f(0, \phi)=0$ for all $\phi$ and assume

A1 $A=D_{x} f(0,0)=\left(\partial f_{i} / \partial x_{j}\right)(0,0)$ is the linearization of system (36) around the equilibrium 0 with $\phi$ evaluated at 0 . Zero is a simple eigenvalue of $A$ and other eigenvalues of $A$ have negative real parts.

A2 Matrix A has a right eigenvector $\mathbf{u}$ and a left eigenvector $\mathbf{v}$ corresponding to the zero eigenvalue.

Let $f_{k}$ be the $k$ th component of $f$ and

$$
a=\sum_{k, i, j=1}^{n} v_{k} u_{i} u_{j} \frac{\partial^{2} f_{k}}{\partial x_{i} \partial x_{j}}(0,0), \quad b=\sum_{k, i=1}^{n} v_{k} u_{i} \frac{\partial^{2} f_{k}}{\partial x_{i} \partial \phi}(0,0) .
$$

The local dynamics of (36) around 0 are totally governed by $a$ and $b$.

(i) $a>0, b>0$. When $\phi<0$ with $|\phi| \ll 1,0$ is LAS and there exists a positive unstable equilibrium; when $0<\phi \ll 1$, 0 is unstable and there exists a negative LAS equilibrium. 
(ii) $a<0, b<0$. When $\phi<0$ with $|\phi| \ll 1,0$ is unstable; when $0<\phi \ll 1$, asymptotically stable and there exists a positive unstable equilibrium.

(iii) $a>0, b<0$. When $\phi<0$ with $|\phi| \ll 1,0$ is unstable and there exists a negative LAS equilibrium; when $0<\phi \ll 1$, 0 is stable and a positive unstable equilibrium appears.

(iv) $a<0, b>0$. When $\phi$ changes from negative to positive, 0 changes its stability from stable to unstable. Correspondingly, a negative equilibrium becomes positive and LAS.

Computations of $a$ and $b$. For system (30), the associated non zero partial derivatives of $\mathbf{F}$ at the DFE are given by,

$$
\begin{gathered}
\frac{\partial^{2} f_{12}}{\partial^{2} x_{13}}=\frac{2(\alpha+\mu) \beta^{*} c_{\mathrm{m}_{2}}}{\Lambda \pi_{2}}, \frac{\partial^{2} f_{12}}{\partial x_{13} \partial x_{15}}=\frac{\partial^{2} f_{12}}{\partial x_{15} \partial x_{13}}=\frac{(\alpha+\mu)(1+\theta) \beta^{*} c_{\mathrm{m}_{2}}}{\Lambda \pi_{2}}, \\
\frac{\partial^{2} f_{12}}{\partial^{2} x_{15}}=\frac{2(\alpha+\mu) \theta \beta^{*} c_{\mathrm{m}_{2}}}{\Lambda \pi_{2}}, \frac{\partial^{2} f_{13}}{\partial^{2} x_{13}}=\frac{-2(\alpha+\mu) \delta_{2} \beta^{*} c_{\mathrm{m}_{2}}}{\Lambda \pi_{2}} \\
\frac{\partial^{2} f_{13}}{\partial x_{13} \partial x_{15}}=\frac{\partial^{2} f_{13}}{\partial x_{15} \partial x_{13}}=\frac{-(\alpha+\mu)(1+\theta) \delta_{2} \beta^{*} c_{\mathrm{m}_{2}}}{\Lambda \pi_{2}}, \frac{\partial^{2} f_{13}}{\partial^{2} x_{15}}=\frac{-2(\alpha+\mu) \theta \delta_{2} \beta^{*} c_{\mathrm{m}_{2}}}{\Lambda \pi_{2}} .
\end{gathered}
$$

From (38) it follows that

$$
\begin{aligned}
a & =\sum_{k, i, j=12}^{15} v_{k} u_{i} u_{j} \frac{\partial^{2} f_{k}}{\partial x_{i} \partial x_{j}}\left(\mathcal{E}^{0}\right)=\frac{-2 v_{13}(\alpha+\mu) \theta \delta_{2} \beta^{*} c_{\mathrm{m}_{2}}}{\Lambda \pi_{2}}\left(u_{13}+u_{15}\right)\left(u_{13}+\theta u_{15}\right) \\
& <0 .
\end{aligned}
$$

From (39), $a<0$ and for the sign of $b$, it is associated with the following non-vanishing partial derivatives of $\mathbf{F}$,

$$
\frac{\partial^{2} f_{12}}{\partial x_{13} \partial \beta^{*}}=-\delta_{2} c_{m_{2}}, \quad \frac{\partial^{2} f_{13}}{\partial x_{13} \partial \beta^{*}}=\delta_{2} c_{m_{2}} .
$$

It follows from expressions in (40) that

$$
b=\sum_{k, i=12}^{15} v_{13} u_{i} \frac{\partial^{2} f_{13}}{\partial x_{i} \partial \beta^{*}}\left(\mathcal{E}^{0}\right)=u_{13} v_{13} \frac{\partial^{2} f_{13}}{\partial x_{13} \partial \beta^{*}}>0 .
$$

Thus $a<0$ and $b>0$ from computations of $a$ and $b$. Using Theorem 1 above item (i) and (iv) we establish the following result Lemma 4.

LEMMA 4. Since $a<0$ and $b>0$, then model system (22) has a transcritical bifurcation at $\mathcal{R}_{\mathrm{t}}=1$, and a unique EE $\left(\mathcal{E}^{*}\right)$ guaranteed by Theorem 1 is LAS for $\mathcal{R}_{\mathrm{t}}>1$ and unstable otherwise.

\section{Summary and concluding remarks}

In this paper, we presented a sex-structured HIV/AIDS model for assessing the effects of homosexuals and bisexuals on the intrinsic dynamics of the disease in heterosexual settings. We extend the model to investigate separately the effects of condom use and 
antiretroviral therapy on controlling HIV/AIDS in a community. The epidemic thresholds for the resulting models are computed and used to assess the effects of condom use and antiretroviral therapy on the transmission dynamics of HIV/AIDS among homosexuals, heterosexuals and bisexuals.

The study shows that the use of male condoms is of significant importance for homosexuals, heterosexuals and bisexuals since it reduces the partial reproductive numbers $\mathcal{R}_{\mathrm{m}_{1}}<\mathcal{R}_{01}$ and $\mathcal{R}_{\mathrm{m}_{2}}<\mathcal{R}_{02}$. The use of both the male and female condom was noted to further reduce HIV/AIDS among the heterosexuals and bisexuals since $\mathcal{R}_{\mathrm{fm}_{2}}<\mathcal{R}_{\mathrm{m}_{2}}<\mathcal{R}_{02}$. The results obtained also suggest that there are certain values of $\mathcal{R}_{0}$ at which the use of condoms will not reduce the reproductive numbers $\mathcal{R}_{\mathrm{fm}_{2}}, \mathcal{R}_{\mathrm{m}_{2}}$ to values below unity even if compliance of $100 \%$ is achieved. Furthermore, for the values of $\mathcal{R}_{0}$ for which $\mathcal{R}_{\mathrm{fm}_{2}}$ and $\mathcal{R}_{\mathrm{m}}$ can be reduced to values below a unity $\left(\mathcal{R}_{\mathrm{fm}}, \mathcal{R}_{\mathrm{m}_{2}}<1\right)$, the compliance may be high and perhaps unattainable, implying that for such populations, HIV/AIDS may not be controlled using female and male condoms only, instead other preventive and therapeutic strategies should be adopted (a similar result obtained in [17]).

We have presented a deterministic compartmental model of HIV/AIDS in a community where those with AIDS symptoms are treated and become sexually active. Similar to $[2,13]$, our results show that the treatment which prolongs the incubation period but does not reduce HIV transmission rate may even enlarge the epidemic. The results show that the treatment with amelioration of AIDS patients will only help the community if the drugs used are $100 \%$ effective in stopping HIV transmission, suggesting that medical researchers should focus more on reducing HIV transmission rates when developing new treatment drugs [13]. Further, treated AIDS patients should not be involved in risk sexual behaviour defined in terms of inconsistent condom use or having multiple sex partners. The possibility of widescale use of the treatment drugs leading to changes in patterns of risk behaviour, particularly a disinhibition effect, has been of considerable concern. There are competing possible effects at individual level, treated AIDS patients may increase the frequency of sexual activity due to the severity of their symptoms decreasing, but may receive effective prevention counselling upon treatment initiation, which would decrease the frequency of risky activities [3]. If drugs used for therapy are $100 \%$ effective and a positive change in the behaviour of treated individuals is achieved, the use of therapeutic drugs will not affect the development of HIV/AIDS in communities but will help communities by lengthening the lives of the infected (reducing morbidity/mortality and socio-economic costs), and such communities would benefit more if people are to know their HIV/AIDS status and if treatment drugs are to be affordable, thus more people would be treated. The treatment with amelioration of AIDS patients should be accompanied with intense public health educational programs which are capable of changing the attitude of treated AIDS patients towards safe sex, since there is no $100 \%$ effective drug in stopping HIV transmission currently in sight. We conclude from the study that the holistic use of the condoms and the treatment as intervention strategies can help contain the HIV/AIDS epidemic and reduce its burden among heterosexuals, homosexuals and bisexuals if implemented strategically in affected communities.

\section{Acknowledgements}

The authors wish to thank anonymous referees for helpful comments which resulted in much improvement of the paper. CC and ZM acknowledge with thanks financial support from the National Science Council of Taiwan. 


\section{References}

[1] R.M. Anderson and R.M. May, Infectious Diseases of Humans, Oxford Univesity Press, London/New York, 1991.

[2] R.M. Anderson, S. Gupta, and R.M. May, Potential of community-wide chemotherapy or immunotherapy to control the spread of HIV-1, Nature (1991), pp. 350-356.

[3] R.F. Baggaley, N.M. Ferguson, and G.P. Garnett, The epidemiological impact of antiretroviral use predicted by mathematical models: A review, Emerg. Themes Epidemiol. 2 (2005), p. 9.

[4] R.F. Baggaley, R.G. White, and M.C. Boily, Systematic review of orogenital HIV-1 transmission probabilities, Int. J. Epidemiol. 37(6) (2008), pp. 1255-1265.

[5] M.C. Boily, R.F. Baggaley, L. Wang, B. Masse, R.G. White, R.J. Hayes, and M. Alary, Heterosexual risk of HIV-1 infection per sexual act: Systematic review and meta-analysis of observational studies, Lancet Infect. Dis. 9 (2009), pp. 118-129.

[6] F. Brauer and C. Castillo-Chavez, Mathematical models in population biology and epidemiology, in Texts in Applied Mathematics Series, Vol. 40, Springer-Verlag, New York, 2001.

[7] J. Carr, Applications Centre Manifold Theory, Springer-Verlag, New York, 1981.

[8] C. Castillo-Chavez and B. Song, Dynamical models of tuberculosis and their applications, Math. Biosci. Eng. 1(2) (2004), pp. 361-404.

[9] C. Castillo-Chavez, Z. Feng, and W. Huang, On the computation of $\mathcal{R}_{0}$ and its role on global stability, 2002 (math.la.asu.edu/chavez/2002/JB276.pdf).

[10] O. Diekmann, J.A.P. Heesterbeek, and J.A.P. Metz, On the definition and computation of the basic reproduction ratio $\mathcal{R}_{0}$ in models for infectious diseases in heterogeneous populations, J. Math. Biol. (1990), pp. 365-382.

[11] C.T. Fang, H.M. Hsu, S.J. Twu, M.Y. Chen, Y.Y. Chang, J.S. Hwang, J.D. Wang, and C.Y. Chuang, Decreased HIV transmission after a policy of providing free access to highly active antiretroviral therapy in Taiwan, J. Infect. Dis. 190(5) (2004), pp. 879-885.

[12] H.W. Hethcote, The mathematics of infectious diseases, SIAM Rev. 42(4) (2000), pp. 599-653.

[13] S.F. Hsu Schmitz, Effects of treatment or/and vaccination on HIV transmission in homosexuals with genetic heterogeneity, Math. Biosci. 167 (2000), p. 118.

[14] T. Lehner and M.A. Chiasson, Seroprevalence of human immunodeficiency virus type 1 and sexual behaviors in bisexual African-American and Hispanic men visiting a sexually transmitted disease clinic in New York City, Am. J. Epidemiol. 147(3) (1998), pp. 269-272.

[15] M.V. Mourao, Comparing risk factors and strategies towards HIV in heterosexual bisexual and gay men attending Portuguese night bars, Int. Conf. AIDS 8 (1992), p. 177, 19-24 July (abstract no. PuC 8148).

[16] Z. Mukandavire and W. Garira, HIV/AIDS model for assessing the effects of prophylactic sterilizing vaccines, condoms and treatment with amelioration, J. Biol. Syst. 14(3) (2006), pp. 323-355.

[17] Z. Mukandavire and W. Garira, Sex-structured HIV/AIDS model to analyse the effects of condom use with application to Zimbabwe, J. Math. Biol. 54(5) (2007), pp. 669-699.

[18] Z. Mukandavire, C. Chiyaka, G. Magombedze, G. Musuka, and N. Malunguza, Assessing the effects of homosexuals and bisexuals on the intrinsic dynamics of HIV/AIDS in African heterosexual settings, Math. Comp. Model. 49(9-10) (2009), pp. 1869-1882.

[19] G. Vijayakumar, Z. Mabude, J. Smit, M. Beksinska, and M. Lurie, A review of female-condom effectiveness: Patterns of use and impact on protected sex acts and STI incidence, Int. J. STD AIDS 17 (2006), pp. 652-659.

[20] P. van den Driessche and J. Watmough, Reproduction numbers and sub-threshold endemic equilibria for compartmental models of disease transmission, Math. Biosci. (2002), pp. 29-48.

[21] WHO Guidelines for HIV Diagnosis and Monitoring of Antiretroviral Therapy, 2005. Available online at: http://www.searo.who.int/linkfiles/publications_HLM-382Rev/.pdf 


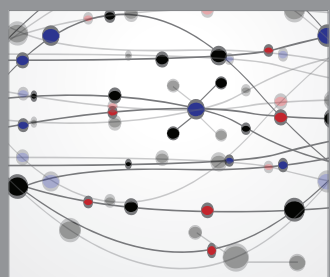

The Scientific World Journal
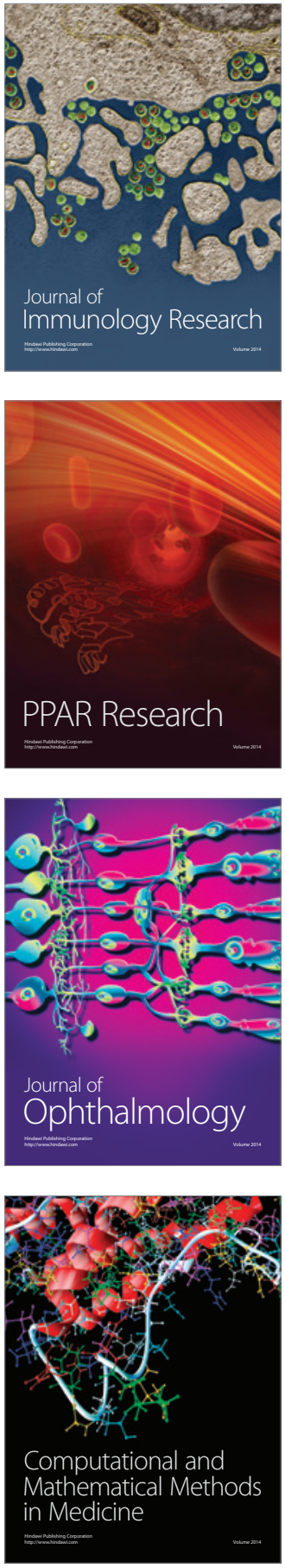

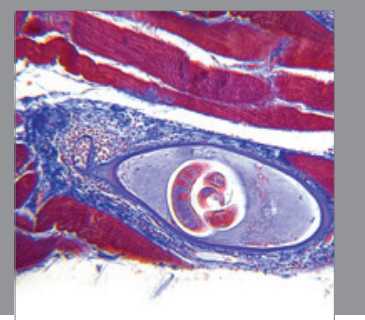

Gastroenterology

Research and Practice
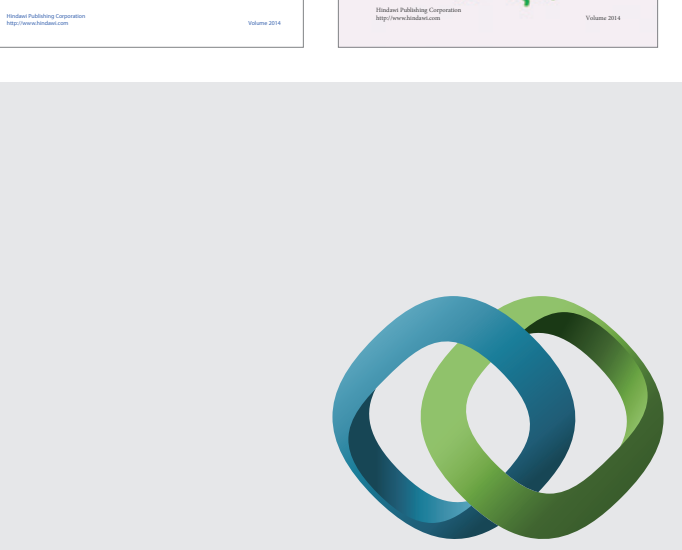

\section{Hindawi}

Submit your manuscripts at

http://www.hindawi.com
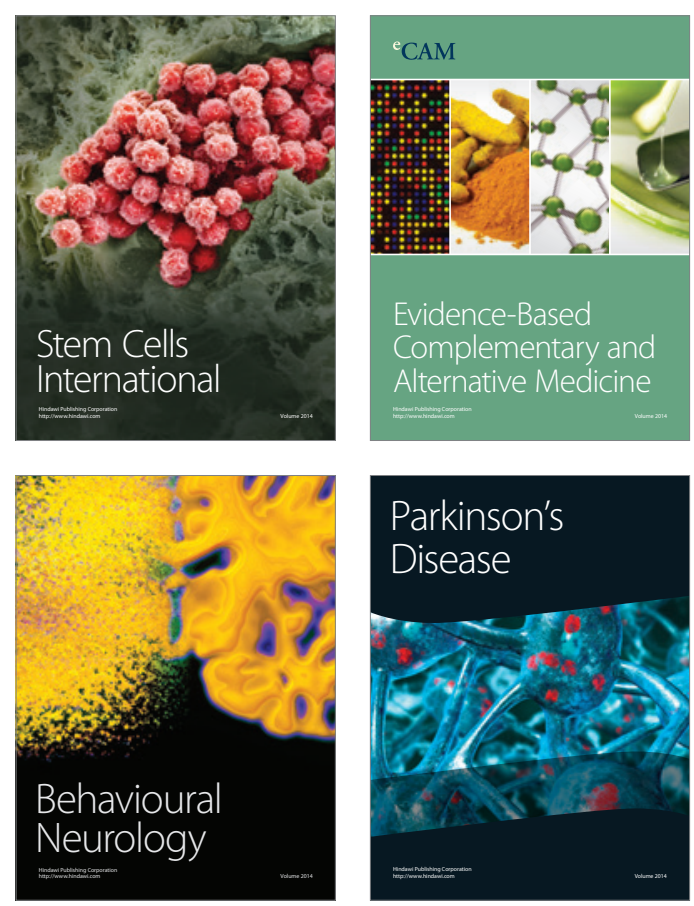

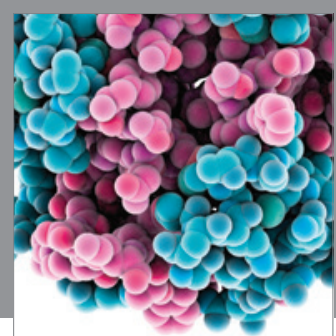

Journal of
Diabetes Research

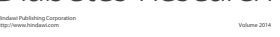

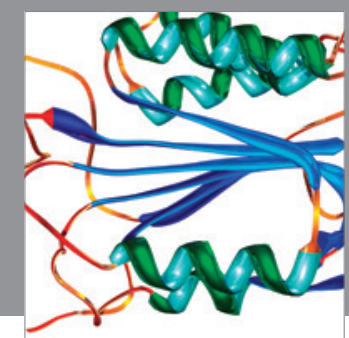

Disease Markers
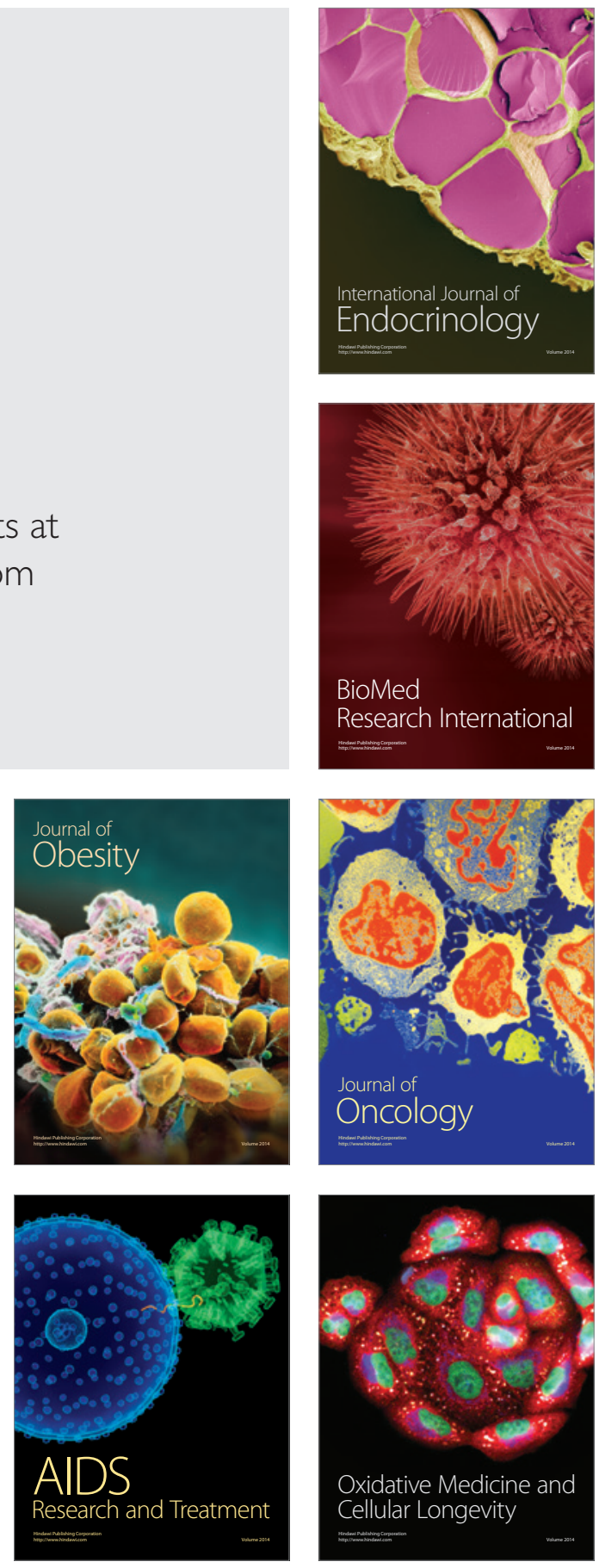